\title{
Downtime Data Analysis Based on Maximum Likelihood (MLE) to Estimate Parameters of Reliability Distributions
}

\author{
Afrah Al-Bossly \\ Department of Mathematics, College of Science and Humanities in Al-Kharj, Prince Sattam Bin Abdulaziz \\ University \\ 11942 Al-Kharj, Saudi Arabia \\ E-Mail: a.basli@psau.edu.sa
}

\begin{abstract}
Reliability analysis techniques are customary standard tools that are used for evaluating the performance of different equipment and devices in order to minimize their downtime. To predict the reliability, life data from a sample that is satisfactorily representative of the equipment should be fitted to the suitable statistical distribution. The parameterized distribution may be used to estimate essential characteristics such as failure rate; and probability at a precise time, as well as system reliability. In the current study, Weibull++/ALTA software package is used as a novel tool to fit the available data set to estimate the best fitted probability density function (PDF) using Maximum Likelihood (MLE) for parameter estimation. The determined distributions are then assessed using goodness-of-fit test to define how well it fits the available data set. There are multiple methods for determining goodness-of-fit. Weibull distributions and their special cases' parameters have an effect on life times.
\end{abstract}

Keywords: Probability Distribution Function, Weibull Distribution, Parameter Estimation, Mean Time Between Failures, Failure Rate, Mean Time To Repair, Downtime and Reliability

\section{Abbreviations}

Aggregate Criterion
Availability
Combined Cycle Power Plants
Cumulative Distribution Function
Condition Monitoring
Correlation Coefficient Test
Down Time
Fisher Matrix Confidence Bounds
Kolmogorov-Smirnov Test
Likelihood Value Test

$\begin{array}{lll}\text { DESV } & \text { Maximum Likelihood } & \text { MLE } \\ \text { A } & \text { Mean Time Between Failure } & \text { MTBF } \\ \text { CCPP } & \text { Mean Time To Failure } & \text { MTTF } \\ \text { CDF } & \text { Mean Time To Repair } & \text { MTTR } \\ \text { CM } & \text { Median Ranks } & \text { MED } \\ \text { CC } & \text { Non-Homogeneous Poisson Process } & \text { NHPP } \\ \text { DT } & \text { Probability Density Function } & \text { PDF } \\ \text { FM } & \text { Pseudo Failure Characteristic } & \text { PFC } \\ \text { K-S } & \text { Rank Regression Analysis X -Axis } & \text { RRX } \\ \text { LHV } & \text { Reliability } & \text { R(t) }\end{array}$




\section{Introduction}

"Reliability Life Data Analysis" means studying and modeling documented data of an equipment to estimate its significant characteristics such as device reliability, failure rate, or mean time to failure (MTTF). Several studies for reliability assessment were; and still are, conducted.

\subsection{Data fitting and Parameters' Estimation}

Weibull analysis is commonly used in Reliability Life Data Analysis. As previously mentioned, to predict reliability of all products in the population, a sample representing the life data should be fitted to suitable statistical distribution. This in turn means that the distribution parameters that most closely fit the data should be estimated using proper statistical techniques. Khodabina and Ahmadabadi introduced the generalized gamma (GG) distribution defining power and logarithmic moments of GG family [1]. The authors presented a new method to estimate the Generalized Gamma distributions had been used in economics by a number of researchers; Jaggia [2], Yamaguchi [3], and Allenby et al [3][4]. To study reliability, Elmahdy analyzed lifetime data using both "Weibull and Lognormal" distributions' models [5] to make comparison the 2 finite mixture distributions, WMD and LMD for modeling mixed survival data sets. The study comprised fitted pdfs, reliability functions and the mean lifetime; among other functions, for the two distributions. Parameters of these mixture models were estimated using expectation-maximization (EM) and Levenberg-Marquardt algorithms. To define the best fit, the authors performed goodness of fit using Kolmogorov-Smirnov (KS), Akaike's Information Criteria (AIC) tests and correlation coefficient. Accurate assessment and prediction of systems reliability is essential for risk management. If feedback data for the considered operation is available, the classical frequents statistical approach is used by building relevant probabilistic parametric models [6]. Then statistical inference of the parameters of the developed models is carried out. After that the fitted models should be validated using statistical criteria and comparing the different competing models. An indication of simple parametric probability distributions that are frequently utilized in reliability was presented by Coolen [7]. The author exhibited the distributions main characteristics and briefly discussed fundamental molds correlated to their suitability for modeling definite reliability scenarios. The exponential-generalized truncated logarithmic (EGTL) distribution is a two-parameter distribution that was introduced [8]. Modeling the reliability of systems, the authors generalized the exponential-logarithmic (EL) distribution using first-order concepts considering the minimum lifetime. The assumption was a system is considered failed if a given number of the components $(\mathrm{k})$ fail and the $\mathrm{k}^{\text {th }}$ smallest value of lifetime replaces the minimum lifetime. Parameters were estimated using; "MLH", Bayesian approach and other methods. The study was used in a number of reliability applications. Fitting distributions indicate finding a mathematical function which adequately represents a data set of a statistical variable which is then belong to a population with a certain probability density function (pdf). Ricci summarized the steps followed in fitting distributions in choosing the distribution function, estimate parameters, evaluate quality of fit, and applying goodness of fit statistical tests [9].The authors used in their study the statistical environment and language R1. The above literature review shows that researchers used a number of statistical distributions to analyze and fit reliability life data such as; generalized gamma (GG) distribution, Weibull mixture distribution (WMD) and lognormal mixture distribution (LMD) for modeling mixed survival data sets. Basic parametric probability distributions are moreover often utilized as part of more complex statistical models, such as mixture models and Bayesian hierarchical models. In the current paper fitting each of the different cause of downtime; using Weibull++/ALTA software package, showed that the best fit was one of the distributions; 2P Exponential, Generalized Gamma, Log Logistic, and lognormal. 


\subsection{Reliability Approaches and Indices}

A general approach to system reliability assessment is to determine a number (or more) of its reliability indices that measure some aspects of system reliability performance such as mean time between failure (MTBF), failure rate $(\chi)$ and mean time to repair (MTTR) [10]. Many studies considered analytic models that are based on Weibull, exponential, uniform, and other distributions. Reliability prediction approach was presented by Denson through analyzing the failure rate model of plastic encapsulated microcircuits. The approach depended upon the product development stages and its related reliability metric [11]. Various reliability prediction methods, their concepts of application, advantages, and disadvantages were investigated by Thakur \& Sakravdia [12]. The classical approach fits equipment failure rates to statistical models [13]; however in the data-mining methodology, it is displayed utilizing a data-mining algorithmdecision tree induction, establishing logical, mathematical, and statistical relations between MTTF and its several factor of impact (equipment conditions, failure history, etc.). As product failure rates are timedependent, they can be considered as time series. Barabady and Kumar [14] applied several statistical distributions containing Weibull, exponential, normal, and log normal distribution to analyze the reliability of a crushing plant, in order to trace the components with low reliability for a specified performance.

\section{Methodology}

The data gathering plays important role in this study to be able to predict the appropriate distribution as the distributions will be used to analyze the failure rate, reliability, availability, and maintainability of the equipment. Hence, the accomplishment of such research depends on the availability of statistical data for the target equipment in addition to the knowledge of statistical modeling and reliability theories. The following subsections present different tools that are used to estimate the reliability of any mechanical or electric component/or system.

\subsection{Basic Concept and Approach for Reliability Analysis}

Accurate assessment of reliability is of vital importance in the study of the performance modern systems. There are several quantitative measures for the reliability of an item. This item can be anything from a small component to a large system. These measures are listed in the following sub-sections.

\subsubsection{Mean time between failures (MTBF)}

MTBF measures the average time equipment is expected to perform prior to the occurrence of an unplanned failure. Testing the system for a period of time $\mathrm{T}$, registering the number of faults $\mathrm{N}$ that is repaired then system testing is resumed. The repair time is extracted from the total test time T. MTBF is then given by equation 1 [15], [16]:

$\mathrm{MTBF}=\frac{T}{N}=\frac{1}{F}($ hours $), \mathrm{F}=$ expected failure rate.

This index is liable to examining inaccuracies, since the system is viewed for just an example of its all-out life. Derivation from the outcome ought to consider these inaccuracies. Hence taking everything into description, the system with the best MTBF is considered the most reliable. 


\subsubsection{Mean time to repair (MTTR)}

MTTR is a measure of time period that is required for the equipment to get back to normal service status when it fails, also known as "Down Time". MTTR is calculated as shown in the following equations [16], [17].

$$
\operatorname{MTTR}=\frac{\varphi t}{\varphi n}
$$

Where: $\varphi \mathrm{t}=$ total outage hours per year. $\varphi n=$ No. of failure per year

$$
\text { Also, } \text { MTTR }=\frac{1}{\mu}
$$

Where $\mu$ =expected repair rate.

\subsubsection{Availability (A)}

Availability is defined as; "percentage of time that equipment is capable of producing its end product at some specified acceptable level". To calculate availability, the machine working time (Up time, UT) and faulty (or being repaired) time (Down time, DT) are first determined. The total period of observation is the sum of UT + DT and availability is ratio of UT/ total period as exhibited in equations $4 \& 5$ [15]]:

$$
\begin{aligned}
& \mathrm{A}=\frac{U T}{U T+D T} \\
& \mathrm{~A}=\frac{M T B F}{M T B F+M T T R}
\end{aligned}
$$

\subsubsection{Reliability $\left(R_{(t)}\right)$}

Reliability is defined as "the capability of apparatus to perform; satisfactorily, the essential function under specified situations through a specified period of time", Ireson et al. [17]. Reliability can also be defined as "the probability that the apparatus is operating without failure in the time period $t$ " as shown in the following equation [17], [18].

$$
\mathbf{R}_{(\mathrm{t})}=e^{\frac{t}{M T B F}}
$$

Using equation (1) in equation (6), we have

$$
R_{(t)}=e^{-F t}
$$

Where; $\mathrm{t}$ = specified failure-free operation period of time.

\subsection{Data Fitting and Parameters' Estimation}

To assess reliability realistic modeling of the system components is required. In practice, not all the assessments of the system under analysis could be complete captured in the model. This is due to the inherently random nature of a number of the phenomena occurring through the system life, and the insufficient information some of them. "Reliability Life Data Analysis" is generally denoted to as "Weibull 
Analysis". To predict life of all products in the population, data from a representative sample is fitted to the suitable statistical distribution, hence the parameters of the statistical distribution that is considered the best fit should be estimated. The "probability density function $(P D F)$ " is a function that; mathematically, pronounces the distribution. The PDF of some statistical distributions are exhibited in the next subsections.

\subsubsection{Lognormal distribution}

The time to failure $\mathrm{T}$ of an item is said to be $\log$ normally distributed with parameters $v$ and $\tau^{2}$,

$\mathrm{T} \sim \operatorname{lognormal}\left(v, \tau^{2}\right)$, if $\mathrm{Y}=\mathrm{In} \mathrm{T}$ is normally (Gaussian) distributed with mean $v$ and variance $\gamma^{2}$ [i.e., $\mathrm{Y}$ $\left.\mathrm{X}\left(v, \tau^{2}\right)\right]$. The probability density function of $\mathrm{T}$ is:

$$
f(t)=\left\{\begin{array}{ll}
\frac{1}{\sqrt{2 \pi t}} e^{-(\ln t-v)^{2} / 2 \tau^{2}} \\
0 \quad \text { otherwise }
\end{array} \text { for } t>0\right.
$$

The lognormal probability density is sketched in Fig. 2.19 for selected values of $v$ and $\tau$.

The mean time to failure is:

$$
\mathrm{MTTF}=e^{v+\tau^{2} / 2}
$$

\subsubsection{Weibull Distribution}

The "3-parameter Weibull pdf" is given by [20], [2]:

$$
f(t)=\frac{\beta}{\eta}\left(\frac{t-\gamma}{\eta}\right)^{\beta-1} e^{-\left(\frac{t-\gamma}{\eta}\right)^{\beta}}
$$

Where:

$$
\begin{aligned}
& f(t) \geq 0, t \geq 0 \text { or } \gamma \\
& \beta>0 \\
& \eta>0 \\
& -\infty<\gamma<+\infty
\end{aligned}
$$

Also,

$\eta=$ "Scale Parameter", that describes where the bulk of the distribution is located.

$\beta=$ "Shape Parameter" (or slope)

$\gamma=$ "Location Parameter" (or failure free life)

The "2- parameter Weibull pdf" is given by [19], [20]:

The 2-parameter Weibull $p d f$ (in this case $\gamma=0$ ), is specified by:

$$
f(t)=\frac{\beta}{\eta}\left(\frac{t}{\eta}\right)^{\beta-1} e^{-\left(\frac{t}{\eta}\right)^{\beta}}
$$

\section{The Mean or MTTF}

The mean, $\bar{T}$, of the Weibull $p d f$ (eq. 11) is also known as MTTF.

$$
\bar{T}=\gamma+\eta \Gamma\left(\frac{1}{\beta}+1\right)
$$

where, $\Gamma\left(\frac{1}{\beta}+1\right)$ is the gamma function assessed at the value of: $\left(\frac{1}{\beta}+1\right)$ 
The gamma function is well-defined as:

$$
\Gamma(n)=\int_{0}^{\infty} e^{-x} x^{n-1} d x
$$

For the 2-parameter case, this can be reduced to:

$$
\bar{T}=\eta \cdot \Gamma\left(\frac{1}{\beta}+1\right)
$$

Note that specific practitioners incorrectly assume that $\eta$ is equal to the MTTF, $\bar{T}$. This is only accurate for the case of: $\beta=1$ or:

$$
\begin{aligned}
\overline{\boldsymbol{T}} & =\boldsymbol{\eta} \cdot \boldsymbol{\Gamma}\left(\frac{\mathbf{1}}{\mathbf{1}}+\mathbf{1}\right) \\
& =\eta \cdot \Gamma(2) \\
& =\eta \cdot 1 \\
& =\eta
\end{aligned}
$$

\section{The Median}

The median, $\breve{T}$ of the Weibull distribution is given by:

$$
\breve{T}=\gamma+\eta(\ln 2)^{\frac{1}{\beta}}
$$

\section{The Mode}

While the mode, $\widetilde{T}$ is:

$$
\widetilde{T}=\gamma+\eta\left(1-\frac{1}{\beta}\right)^{\frac{1}{\beta}}
$$

\section{The Standard Deviation}

The standard deviation $\sigma_{T}$ is assumed by:

$$
\sigma_{T}=\eta \cdot \sqrt{\Gamma\left(\frac{2}{\beta}+1\right)-\Gamma\left(\frac{1}{\beta}+1\right)^{2}}
$$

\section{Weibull Reliability Function}

3-parameter Weibull cumulative density function, $c d f$, is given by equation 15 :

$$
F(t)=1-e^{-\left(\frac{t-\gamma}{\eta}\right)^{\beta}}
$$

Some authors refer to the above equation as unreliability $Q(t)$.

As the reliability function of a distribution equals one minus the $c d f$, the 3-parameter Weibull distribution' reliability function is:

$$
R(t)=e^{-\left(\frac{t-\gamma}{\eta}\right)^{\beta}}
$$

\section{Weibull Conditional Reliability Function}


The reliability function of 3-parameter Weibull conditional reliability is:

$$
\begin{array}{r}
R(t \mid T)=\frac{R(T+t)}{R(T)}=\frac{e^{-\left(\frac{T+t-\gamma}{\eta}\right)^{\beta}}}{e^{-\left(\frac{T-\gamma}{\eta}\right)^{\beta}}} \\
\text { Or: } R(t \mid T)=e^{-\left[\left(\frac{T+t-\gamma}{\eta}\right)^{\beta}-\left(\frac{T-\gamma}{\eta}\right)^{\beta}\right]}
\end{array}
$$

It is called conditional because reliability of a novel assignment is calculated built on the detail that the unit (or units) has already collected hours of operation successfully.

\section{The Weibull Failure Rate Function}

Equation (19) represents the "Weibull failure rate function", $\lambda(t)$

$$
\lambda(t)=\frac{f(t)}{R(t)}=\frac{\beta}{\eta}\left(\frac{t-\gamma}{\eta}\right)^{\beta-1}
$$

\subsubsection{Gamma distribution}

The gamma distribution may be considered as a generalized case of the exponential distribution with mean= $1 / \lambda, \lambda>0$. The mean $=1 / \lambda$, signifies the time elapsed till the first event takes place, where $\lambda$ is the mean of the Poisson process that generate the events. While the waiting time until the $\mathrm{a}^{\text {th }}$ event to occur is represented by gamma random variable $X$. Hence,

$$
\mathrm{X}=\sum_{i}^{a} Y
$$

Where $Y 1, \ldots ; Y n$ are "independent exponential random variables" with mean $=1 / \lambda$.

The probability density function of Gamma distribution is given by (21):

$$
f(x ; \alpha, \beta)=\frac{1}{\Gamma(\alpha) \beta^{\alpha}} e^{-x / \beta} x^{\alpha-1}, x>0, \alpha>0, \beta>0
$$

Where $\alpha$ is the "shape parameter", $\beta$ is the "scale parameter", and $\Gamma$ is the "gamma function".

\subsubsection{G-Gamma distribution}

Generalized gamma (GG) distribution that is considered a flexible distribution, and has subfamilies; "exponential, gamma, and Weibull", and a limiting distribution "lognormal distribution" [1].

X-Generalized gamma $(\alpha, \beta, \gamma)$ representation is applied to specify the generalized gamma distribution with real positive parameters; scale parameter $\alpha$, while $(\beta \& \gamma)$ are the shape parameters. The probability density function of generalized gamma random variable $X$ has as following in equation (22).

$$
f(x)=\frac{\gamma x^{\gamma \beta-1} e^{-(x / \alpha)^{\gamma}}}{\alpha^{\gamma \beta} \Gamma(\beta)}, x>0
$$

The median time to failure satisfying $R(\mathrm{tm})=0.51$ is 


$$
\begin{aligned}
& \boldsymbol{R}\left(\boldsymbol{t}_{\boldsymbol{m}}\right)=\mathbf{0 . 5} \\
& \boldsymbol{t}_{\boldsymbol{m}}=\boldsymbol{e}^{v} \text { and the mode of the distribution is } \\
& t_{\text {mode }}=e^{v-\tau^{2}}
\end{aligned}
$$

\subsubsection{The Loglogistic Distribution}

The $\log \operatorname{logistic}$ distribution is a 2-parameter distribution with parameters $\mu$ and $\sigma$. Equation (22) gives the $p d f$ for this distribution:

$$
f(t)=\frac{e^{2}}{\sigma t\left(1+e^{z}\right)^{2}}
$$

where:

$$
\begin{aligned}
& z=\frac{\dot{t}-\mu}{\sigma} \\
& \dot{t}=\ln (t)
\end{aligned}
$$

and: $\mu=$ scale parameter

$$
\sigma=\text { shape parameter }
$$

where $\mathbf{0}<\boldsymbol{t}<\infty,-\infty<\boldsymbol{\mu}<\infty$ and $\mathbf{0}<\boldsymbol{\sigma}<\infty$

\section{The Loglogistic Reliability Function}

The reliability for an operation of time $T$, starting at 0 , following the log logistic distribution is determined by:

$$
R=\frac{1}{1+e^{z}}
$$

where:

$$
\begin{aligned}
z & =\frac{t-\mu}{\sigma} \\
t & =\ln (t)
\end{aligned}
$$

\section{The "log logistic Failure Rate Function"}

The log logistic failure rate is given by:

$$
\lambda(t)=\frac{e^{z}}{\sigma t\left(1+e^{z}\right)}
$$

\subsubsection{The 2-Parameter Exponential Distribution}

The $p d f$ of the "2-parameter exponential" is given by:

$$
f(t)=\lambda e^{-\lambda(t-\gamma)}, f(t) \geq 0, \lambda>0, t \geq \gamma
$$

Where: $\gamma$ is the location parameter.

Specific of the characteristics of the 2-parameter exponential distribution are discussed in Kececioglu [21, 22]: 
- Positive location parameter; $\gamma$, shifts the beginning of the distribution right of the origin by a distance $\gamma$. This means that failures possibility starts only after $\gamma$ hours of operation.

- The "scale parameter" is: $\frac{\mathbf{1}}{\lambda}=\boldsymbol{t}-\boldsymbol{\gamma}=\boldsymbol{m}-\boldsymbol{\gamma}$

- The distribution starts at $\boldsymbol{t}=\boldsymbol{\gamma}$ at the level of $\boldsymbol{f}(\boldsymbol{t}=\boldsymbol{\gamma})=\boldsymbol{\lambda}$, decreasing exponentially and monotonically thereafter as $t$ increase beyond $\gamma$.

- $\quad$ As $t \rightarrow \infty, f(t) \rightarrow 0$

\section{The Mean or MTTF}

The mean $\bar{T}$ or (MTTF) is given by:

$$
\begin{gathered}
\bar{T}=\int_{\gamma}^{\infty} t \cdot f(t) d t \\
=\int_{\gamma}^{\infty} t \cdot \lambda \cdot e^{-\lambda t} d t \\
\bar{T}=\gamma+\frac{1}{\lambda}=m
\end{gathered}
$$

It could be noted that MTTF is the inverse of the constant failure rate of the exponential distribution.

\section{The Median}

The median $\breve{T}$ is:

$$
\breve{T}=\gamma+\frac{1}{\lambda} .0 .693
$$

\section{The Mode}

The mode $\tilde{T}$ is:

$$
\widetilde{T}=\gamma
$$

The Standard Deviation

The standard deviation $\sigma_{T}$ is:

$$
\sigma_{T}=\frac{1}{\lambda}=m
$$

\section{The Exponential Reliability Function}

The equation for the 2-parameter exponential cumulative density function, or $c d f$, is given by:

$$
F(t)=Q(t)=1-e^{-\lambda(t-\gamma)}
$$

As the reliability function of a distribution equals one minus the $c d f$, then reliability function of the 2parameter exponential distribution is as follows:

$$
\begin{aligned}
& R(t)=1-Q(t)=1-\int_{0}^{t-\gamma} f(x) d x \\
& R(t)=1-\int_{0}^{t-\gamma} \lambda e^{-\lambda x} d x=e^{-\lambda(t-\gamma)}
\end{aligned}
$$

The "1-parameter exponential reliability function" is given by: 


$$
R(t)=e^{-\lambda t}=e^{-\frac{t}{m}}
$$

\section{The Exponential Conditional Reliability Function}

The equation of "exponential conditional reliability" expresses the reliability of an operation with a duration $t$ that accumulated $T$ hours till a new $t$ mission starts. The "exponential conditional reliability function" is:

$$
R(t \mid T)=\frac{R(T+t)}{R(T)}=\frac{e^{-\lambda(T+t-\gamma)}}{e^{-\lambda(T-\gamma)}}=e^{-\lambda t}
$$

This means that the reliability for a assignment of $\mathrm{t}$ period started after the constituent has already accrued $\mathrm{T}$ hours of process from age zero are only a function of the assignment period, and not the age function at the beginning of the assignment. This is known as the memory less property.

\section{The Exponential Failure Rate Function}

The exponential failure rate function is:

$$
\lambda(t)=\frac{f(t)}{R(t)}=\frac{\lambda e^{-\lambda(t-\gamma)}}{e^{-\lambda(t-\gamma)}}=\lambda=\text { constant }
$$

\section{CASE STUDY}

To estimate the reliability, failure, and probability density functions of machines in a production facility, data are obtained from a previous study in Coldair Engineering Company for refrigerators and air conditioners in Sudan [23]. The available data are monthly down- time summery reports for 6 months of 2015 downtime causes of machines producing required plastic parts. After fitting these data to the suitable distribution of each cause of downtime; estimating their parameters, both failure and reliability versus time relations are then estimated. A sample of this data is exhibited in Table 1, while the other set of data are

\begin{tabular}{|c|c|c|}
\hline Machine & Downtime & Hour \\
\hline \multirow[t]{6}{*}{ GIESS I } & Machine & 21 \\
\hline & Air Drop & 2 \\
\hline & NSE POWER OFF & 4 \\
\hline & Tools Change & 33 \\
\hline & Heating UP & 5 \\
\hline & MAINTENANCE & 38 \\
\hline \multirow[t]{6}{*}{ GIESS II } & Air Drop & 10 \\
\hline & NSE POWER OFF & 4 \\
\hline & Tools Change & 44 \\
\hline & Heating UP & 3 \\
\hline & MAINTENANCE & 24 \\
\hline & Others & 2 \\
\hline \multirow[t]{3}{*}{ BM BIRAGHI 100} & NSE POWER OFF & 29 \\
\hline & Tools Change & 8 \\
\hline & Heating UP & 32 \\
\hline \multirow[t]{5}{*}{ BM BIRAGHI 720} & NSE POWER OFF & 7 \\
\hline & Tools Change & 9 \\
\hline & Heating UP & 30 \\
\hline & STOCKING & 1 \\
\hline & Others & 1 \\
\hline \multirow[t]{3}{*}{ BM BIRAGHI 125} & NSE POWER OFF & 26 \\
\hline & Tools Change & 10 \\
\hline & Heating UP & 33 \\
\hline \multirow[t]{3}{*}{ BM BIRAGHI 720 NEW } & NSE POWER OFF & 16 \\
\hline & Tools Change & 20 \\
\hline & Heating UP & 46 \\
\hline
\end{tabular}
shown in reference [23]. The tables show the cause of the machine stopping and its duration in hours.

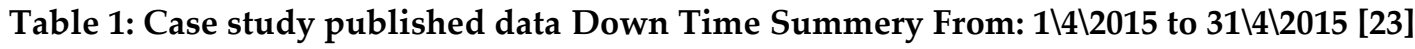




\subsection{Procedures of Weibull++ ALTA Software Application}

To predict the reliability performance of an item or component, available life data; down time or failure time is analyzed and fitted to an adequate statistical distribution. Thus it would be possible to estimation of numerous life characteristics, such as probability of failure, reliability, mean life, or failure rate. The software comprises a number of statistical distributions and analysis approaches [31] such as:

- $\quad 1,2$ and 3 parameter Weibull

- 1 and 2 parameter Exponential

- Normal and Lognormal

- 2, 3 and 4 subpopulation Mixed Weibull

(Situations when there are different trends in the data

and no distinct failure mode could be identified).
- Logistic and Log logistic

- Bayesian-Weibull

- Gamma and Generalized Gamma

All above distributions were fitted to down time data (DT) as shown the following section.

\subsection{Goodness of Fit Tests}

Goodness-of-fit test is then applied on the probability distribution model of the fitted data (in our case it is down time).There is several approaches for defining goodness-of-fit. Several of the utmost prevalent methods utilized contain the chi-square, the "Kolmogorov-Smirnov test", the "Anderson-Darling test", and the "Stephens, M. A. test" [24]. In the current study, DT data for different causes of machine stoppage for 5 months are used as input data, as shown in table 1.

To determine the best fit the software package performs three "goodness of fit tests" that rank the fitted distributions; these tests are:

- The Kolmogorov-Smirnov test (K-S): tests for statistical difference between the expected and results obtained from the fitted distribution.

- The correlation coefficient test (CC): measures how well the plotted points fit a straight line.

- The Likelihood Value test (LHV): calculates the value of the log-likelihood function assumed the parameters of the distribution.

\subsection{Parameter Estimation}

To estimate the reliability of each machine, the reliability function of the cause-of-downtime fitted distribution is used once the distribution parameters are estimated. There are several methods for parameter estimation that can be used such as: the maximum likelihood estimation (MLE) method, rank regression analysis, median ranks (MED), and Fisher matrix confidence bounds (FM). Using rank regression analysis regression line is fitted to the data points to obtain the distribution parameters. The closer regression lines to the plotted points, the better the fit. While maximum likelihood estimation (MLE) method obtains the solution using the likelihood function. Reliability of the entire machine and subsystems is then estimated using best fitted distribution and estimated parameters, as shown in section 4 of results 


\section{RESULTS AND DISCUSSION}

\subsection{Best Fit Distribution}

Using "Weibull++/ ALTA", components' data; shown in table 1 were first fitted using both MLE and RRX and it gave the same results. The resulted distributions are tested using K-S goodness of fit test, Correlation Coefficient (CC) test and Likelihood Value (LHV) test. Based on the three rankings; resulted from the three above mentioned tests, and on weights assigned to the individual criteria, an aggregate criterion (DESV) is applied to choose the best-fitting probability distribution. The described DESV aggregate criterion is shown in Equation 33 [24]. In this study, the used weights are the default values of weights selected by the software "Weibull++/ ALTA" manufacturer. These values resulted from many analyses conducted in applications based on engineering practice. The method assumes that the lowest DESV value corresponds to the best-fitting theoretical distribution. Using down time data, a sample of the results of this ranking procedure of one of the components; air drop is shown in table 2.The results for the other components are illustrated in appendix B. To rank the distributions, the three tests (K-S, CC, and LHV) are used in conjunction with their weights in the following equation to calculate aggregate criterion (DESV) where the distributions are ranked according to its value (rank 1 is that of minimum DESV value) [24 and 32]:

DESV $=($ K-S Rank $\times$ K-S Weight $)+($ CC Rank $\times$ CC Weight $)$ $+($ LKV Rank $\times$ LKV Weight)

A summary of the resulted ranks of one of the causes of machine breakdown in Coldair Company; air drop, is presented in Tables 2. The results for Group2\&3 are exhibited appendix A.

Table 2: DESV Results of Down time fitted Data using MLE Method

\begin{tabular}{|l|l|l|l|l|l|}
\hline Reason of failure & Distribution & RAVGOF & RAVPLOT & RLKV & DESV \\
\hline NSE Power Off & Lognormal & 4 & 1 & 1 & 220 \\
\hline Others & 3P-Weibull & 3 & 2 & 3 & 290 \\
\hline Stocking & 3P-Weibull & 7 & 1 & 1 & 340 \\
\hline Maintenance & 3P-Weibull & 3 & 8 & 3 & 350 \\
\hline \multirow{2}{*}{ Electric } & Gamma & 3 & 1 & 4 & 330 \\
\cline { 2 - 7 } & G-Gamma & 4 & 2 & 3 & 330 \\
\hline Tools Change & G-Gamma & 2 & 2 & 2 & 200 \\
\hline Heating Up & G-Gamma & 1 & 1 & 1 & 100 \\
\hline Machine & Loglogistic & 1 & 1 & 3 & 200 \\
\hline Air Drop & 2P-Exponentional & 3 & 6 & 1 & 230 \\
\hline
\end{tabular}

In the above table: $\mathrm{RAVGOF}=\mathrm{K}-\mathrm{S}, \mathrm{RAVPLOT}=\mathrm{CC}, \mathrm{RLKV}=$ ranking of the LKV test

From table 2, the presented analysis of the down time of the machines due to air drop shows that the bestfitting distribution, according to the aggregate criterion, is the 2P-Exponentialdistribution. Nevertheless, with successive failures, different distribution may be indicated as the best-fit.

\subsection{Characteristics of Each Cause of Breakdown}

Data analysis of the breakdown causes (reasons of failure; listed in the first column of table 2) were found to follow one of these statistical distributions: Lognormal, 3-P Weibull, Gamma, and Generalized Gamma distributions. The characteristics of each of these distributions are presented in the following subsections. 


\subsubsection{Lognormal Distribution}

\section{i) Lognormal distribution characteristics [25]}

The "lognormal distribution" is appropriate to random variates constrained by zero and a few very large values. In this case the distribution is "asymmetrical" and "positively skewed" [25 and 33]. The $p d f$ increases from zero, till it reaches the "mode" then it decreases afterwards. For given $\mu$, the degree of skewness increases with the increase of $\sigma_{T}$, while For the same $\sigma_{T}$, the skewness increases with the increase of $(\dot{\mu}) . \dot{\mu}$ is the mean of the natural logarithms of the time to failure which is the scale parameter, and $\sigma_{T}$ is the standard deviation which is the shape parameter. Another characteristic is that if $\sigma_{T}$ is considerably $>1, p d f$ sharply raise at the beginning.

\section{ii) Cause of breakdown following Lognormal distribution (NSE power off)}

Using ReliaSoft's software, the parameters repaid for the lognormal distribution are continuously logarithmic. Hence, as mentioned above (i), $\dot{\mu}$ signifies the mean of the natural logarithms of the times-tofailure, while $\sigma$ signifies the standard deviation of these data point logarithms. This means the value of the times-to-failure are not utilize as a parameter. Figures 1, 2 and 3 characterize the probability density function ( $p d f)$, Failure rate vs. Time, and Reliability vs. Time, of NSE Power off cause of failure.

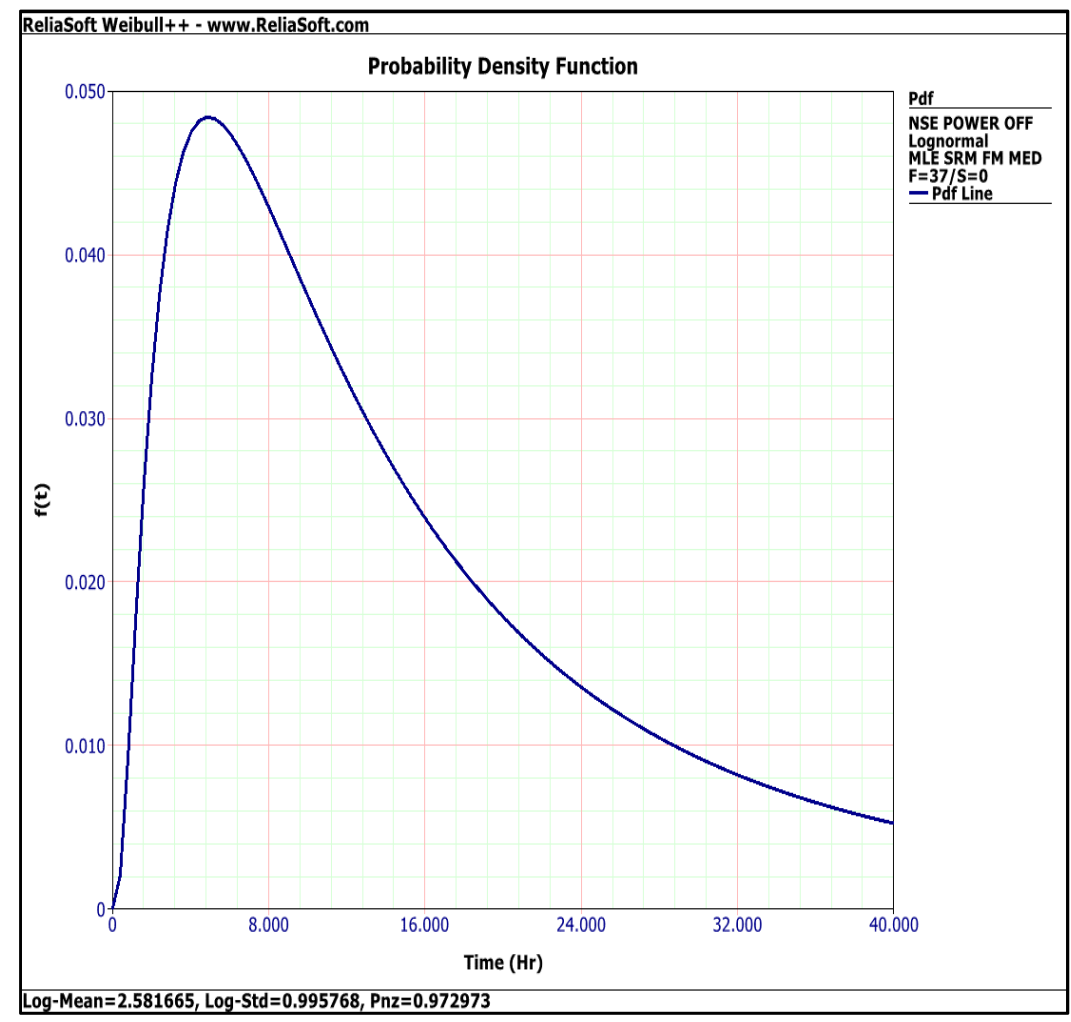

Figure 1: Probability density function $(p d f)$ of NSE Power off (Lognormal) 


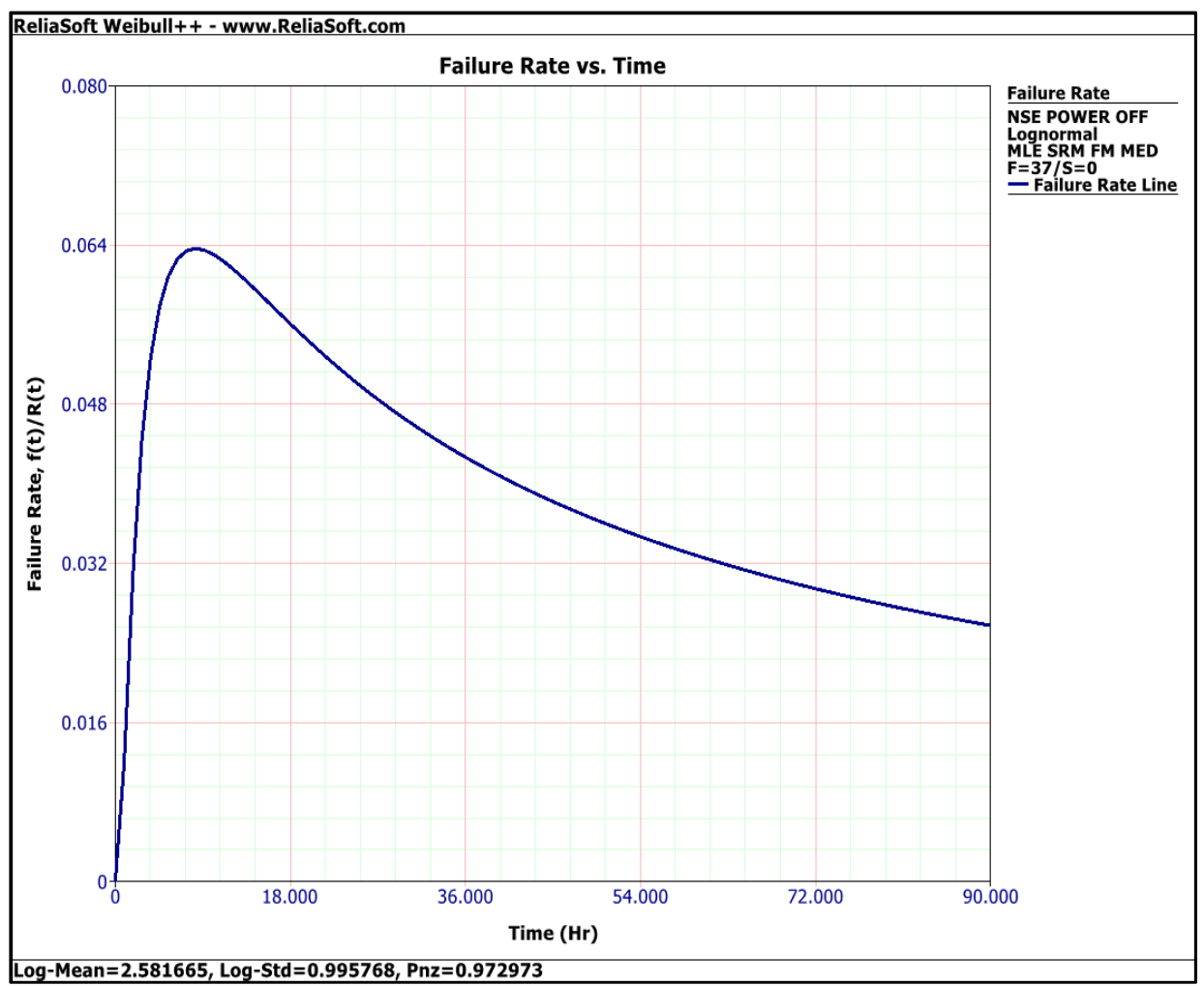

Figure 2: Failure rate vs. Time of NSE Power off (Log normal)

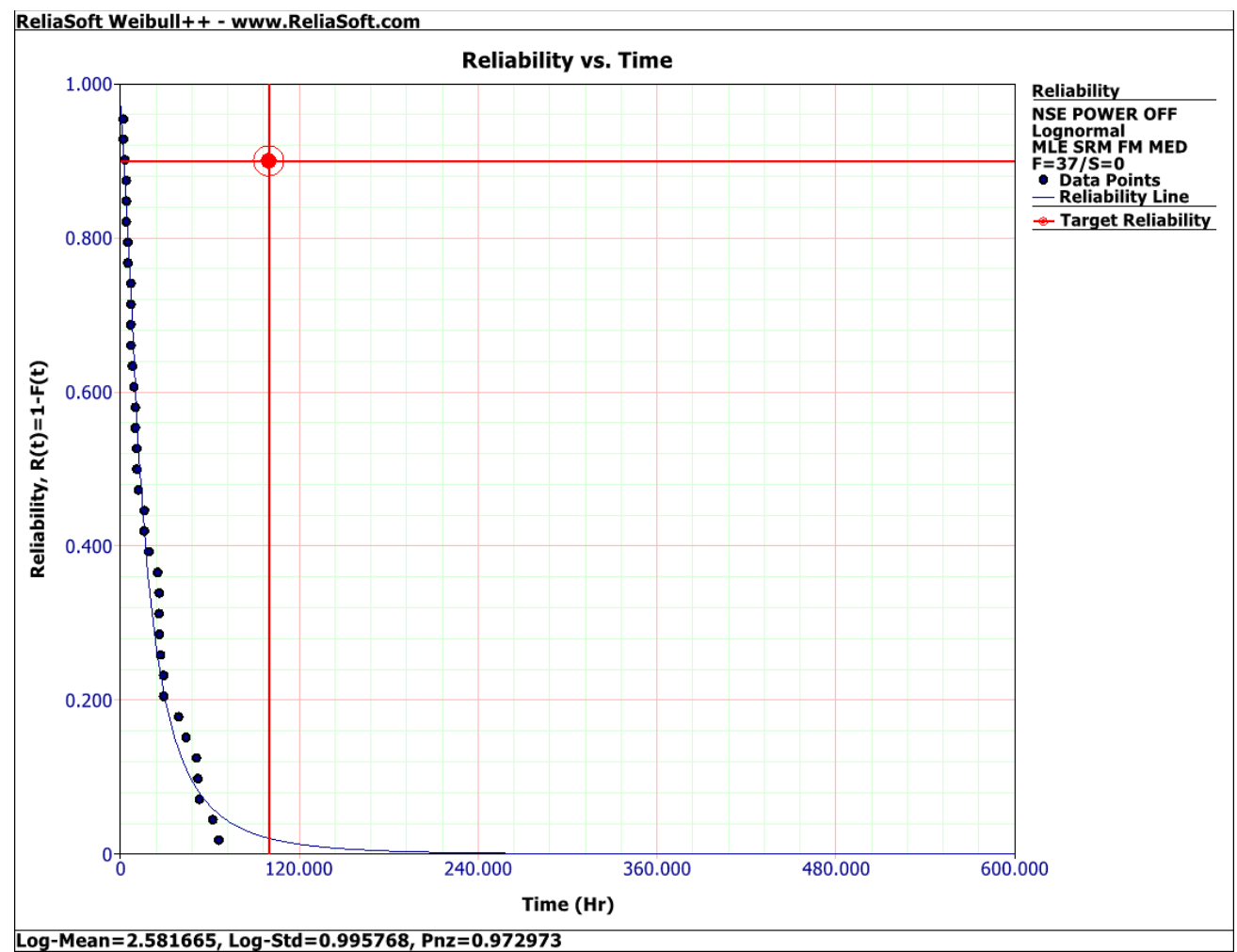

$\underline{L o g}-$ Mean $=2.581665$, Log-Std $=0.995768$, Pnz $=0.972973$

Figure 3: Reliability vs. Time of NSE Power off (Log normal) 
It could be seen in figure 1 that $\dot{\sigma}=0.995768$ (slightly $<1)$, and the mode $(\log$ mean $)=2.581665$, hence pdf begins decreasing directly after the mode.

In figure 3 it is seen that the reliability is $90 \%$ at the beginning of operation $(t=0)$ decreasing rapidly to about $28 \%$ after $24 \mathrm{~min}$ of operation to reach zero after $168 \mathrm{~min}$.

\subsubsection{Weibull Distribution}

\section{i) Weibull distribution characteristics}

"Weibull distribution" is utilized a model variety of life behaviours according to the values of the parameters. The values of the "shape parameter $\beta$ " and the "scale parameter $\eta$ ", affect distribution characteristics; the curve shape, the reliability and the failure rate. The most general form "3-parameter Weibull distribution" is considered. The other forms, such as the 2-parameter distribution (Location parameter $\gamma=0$ ) or the 1-parameter form $(\beta=C=$ constant, can be easily determined [27 and 34].

\section{The Effect of beta on the pdf}

The value of Weibull "shape parameter"; $\beta$ equals the slope of the regressed line in probability plots. Diverse values of"; $\beta$ can change the distribution behaviour. Some values of $\beta$ would cause the distribution equations to convert to those of other distributions. If $\beta=1$, the $p d f$ of the "3-parameter Weibull distribution" changes to that of the "2-parameter exponential distribution" or:

$$
f(t)=\frac{1}{\eta} e^{-\frac{t-\gamma}{\eta}}
$$

Where: $\frac{1}{\eta}=\lambda=$ failure rate. The parameter $\beta$ is a pure number. The Figure 4 shows the effect of different values of $\beta$, on the shape of the $p d f$. It could be seen that $p d f$ shape can take on a variety of forms based on the value of $\beta$.

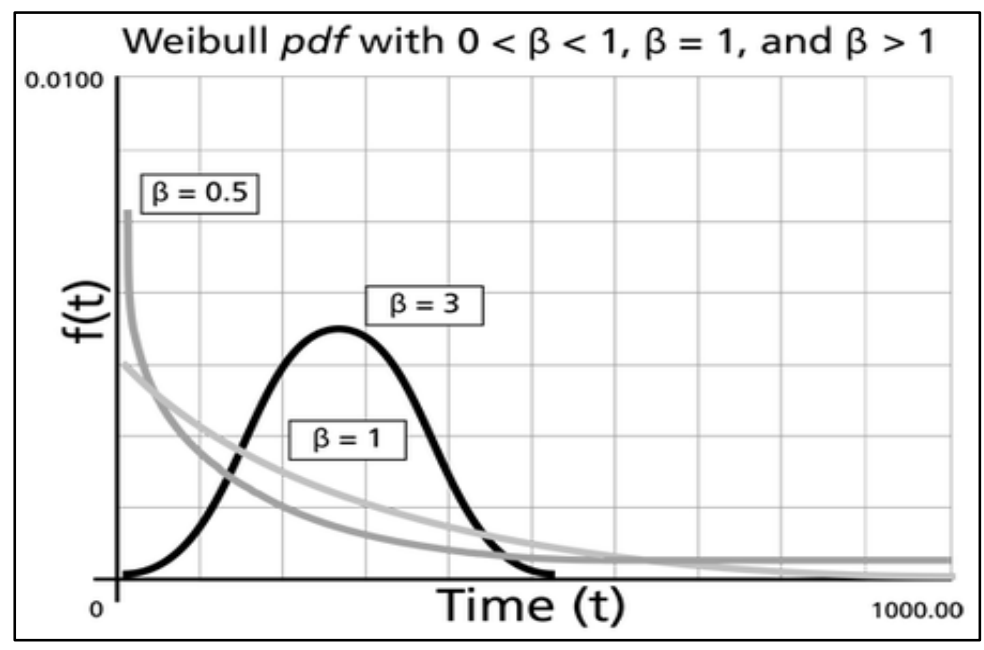

Figure 4: Effect of $\boldsymbol{\beta}$ on Weibull $p d f$ [27]

For: $0<\beta \leq 1$

- As $t \rightarrow 0($ or $\gamma), f(t) \rightarrow \infty$

- As $t \rightarrow \infty, f(t) \rightarrow 0$

- No mode exists. 
For: $\beta>1$

- $f(t)=0$ at $t=0($ or $\gamma)$

- $f(t)$ Increases as $t \rightarrow \tilde{T}$ then it decreases.

- For $\beta<2.6$ the Weibull $p d f$ has a right tail (positively skewed) while for $2.6<\beta<3.7$ there is no tail (coefficient of skewness is almost zero). In case of $\beta>3.7$ a left tail exists (the curve is negatively skewed). Hence, different values of $\beta$ affect both reliability and failure rate functions as for $\beta=0.999, f(0)=\infty$, while for $\beta=1.001, f(0)=0$. This sudden shift is why MLE estimation is complicated when $\beta$ is close to 1, [28].

\section{The Effect of the shape parameter $\beta$ on the Weibull Failure Rate}

Figure 5 exhibits the Effect of $\beta$ on Weibull Failure Rate [27, 28 and 29].

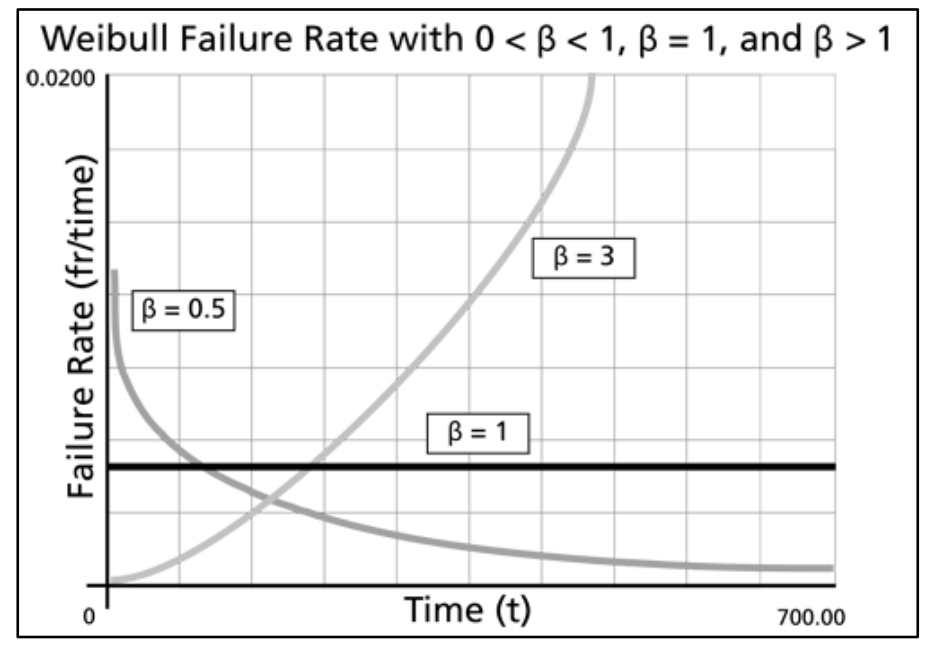

Figure 5: Effect of $\boldsymbol{\beta}$ on Weibull Failure Rate [27]

As indicated in figure 5 , downtime data with $\beta<1$ exhibit a failure rate that decreases with time, while that with $\beta=1$ have a constant failure rate (consistent with the exponential distribution) and populations with $\beta>1$ have a failure rate that increases with time. This means that all three life stages of reliability can be modelled with the Weibull distribution. After that failure rate, $\lambda(t)$ monotonically decreases, approaching the value of zero as $t \rightarrow \infty$ or $\lambda(\infty)=0[27,28$ and 29]. For $\beta=1, \lambda(t)$, yields a constant value of $\frac{1}{\eta}$ or: $\lambda(t)=\lambda=\frac{1}{\eta}$

For $\beta=2$ : a straight line relationship between $\lambda(t)$ and $t$ emerges, starting at a value of $\lambda(t)=0$ at $t=\gamma$, and increasing thereafter with a slope of $\frac{2}{\eta^{2}}$. Consequently, the failure rate increases at a constant rate as $t$ increases. Furthermore, if $\eta=1$ the slope becomes equal to 2 , and when $\beta=0, \lambda(t)$, becomes a straight line which passes through the origin with a slope of 2 . Note that at $=2$, the Weibull distribution equations reduce to that of the Rayleigh distribution.

When $\beta>2$ the $\lambda(t)$ curve is convex, the curve slope increases ast increases. Consequently, the failure rate increases at an increasing rate as $t$ increases, indicating wear out life.

The Effect of $\beta$ on Reliability plot 
- $R(t)$ Decreases sharply and monotonically for $0<\beta<1$ and is convex.

- $F o r \beta>1, R(t)$ decreases as $\beta$ increases. Figure 6 shows the effect of $\beta$ on Weibull Reliability plot

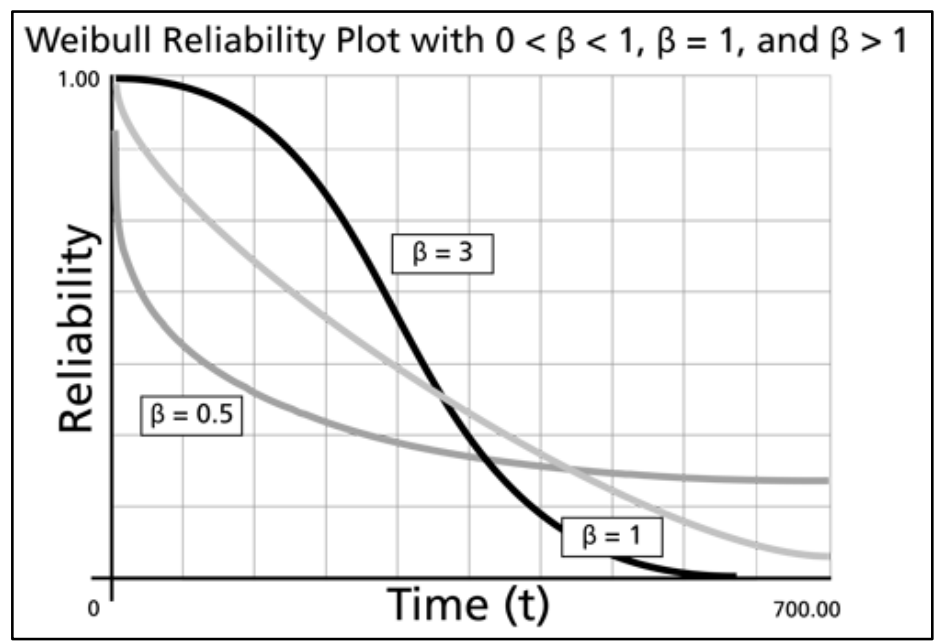

Figure 6: Effect of $\beta$ on Weibull Reliability plot [27]

From figure 6 it is seen that for $\beta=0.5(<1)$, reliability decrease fast. For $\beta=1$, the reliability declines from $100 \%$ at the beginning of operation to reach $3.5 \%$ after $700 \mathrm{hrs}$. In case of $\beta>1$, reliability decreases slowly from $100 \%$ to about $50 \%$ then faster till it reaches 0 .

\section{- The Effect of the scale parameter $\eta$ on Weibull pdf}

A change in the scale parameter $\eta$ has the same effect on the distribution as of changing the $x$-axis scale. If $\eta$ is increased; while holding $\beta$ constant, this will cause the expansion of the $p d f$. Then the zone below a $p d f$ curve is a constant assessment of one, the "peak" of the $p d f$ curve will moreover decrease with the increase of $\eta$, as indicated in figure 7 .

- If $\eta$ is increased while $\beta$ and $\gamma$ are similar, the distribution becomes stretched out to the right and its height decreases, while keeping its shape and location.

- If $\eta$ is decreased while $\beta$ and $\gamma$ are reserved similar, the distribution becomes pressed in towards the left (i.e., towards its beginning or towards 0 or $\gamma$ ), and its height increases.

- $\eta$ has the similar units as $t$, such as hours, miles, cycles, actuations, etc. 


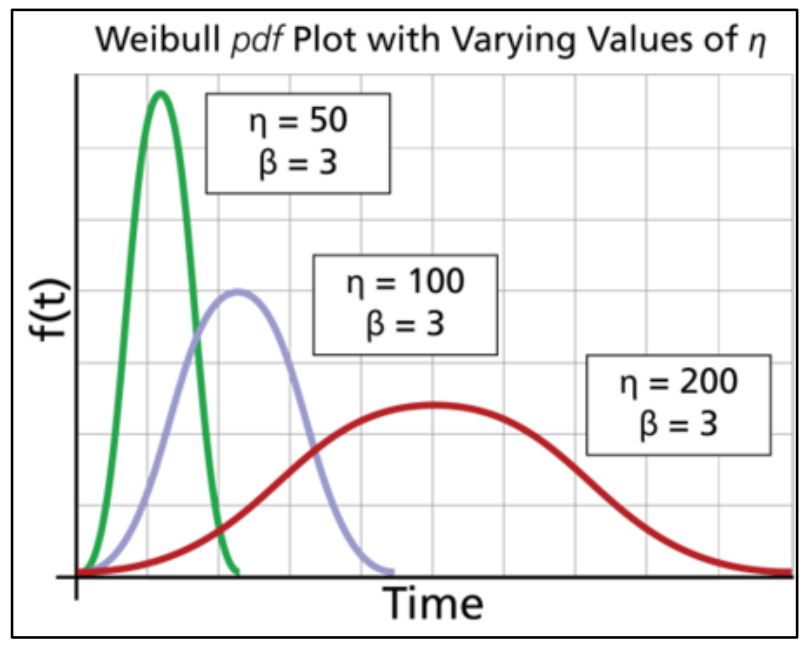

Figure 7: Weibull $p d f$ plot for different values of Scale Parameter $(\boldsymbol{\eta})[27]$

\section{- Effects of the location parameter $\gamma$}

It is obvious that the parameter, $\gamma$, positions the distribution along the time axis. As the value of $\gamma$ changes, the distribution; and its associated function, slide either to the right (if $\gamma>0$ ) or to the left (if $\gamma<0$ ). The parameter $\gamma$ has the same units of the $\mathrm{x}$-axis. Figure 8 illustrates the effect of the location parameter $\gamma$ on Weibull distribution.

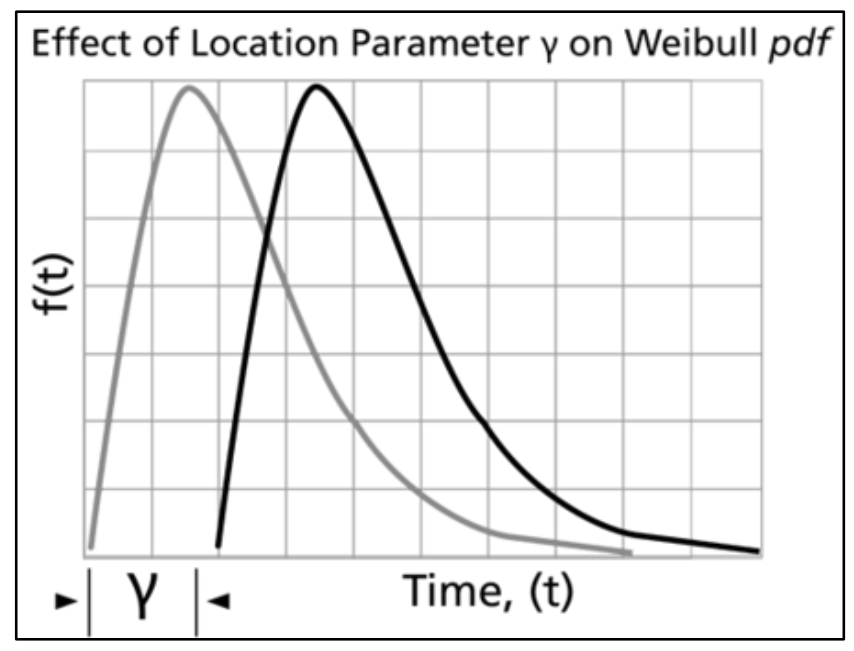

Figure 8: Effect of the location parameter $\gamma$ on Weibull distribution [27]

From figure 8 it is noted that:

- When $\gamma=0$ the distribution starts at $t=0$ or at the origin.

- If $\gamma>0$ the distribution starts at the right of the origin (at distance $=$ ).

- If $\gamma<0$ the distribution starts the left of the origin (at distance $=\gamma$ ).

- A negative $\gamma$ probably signifies that failures had occurred prior to the test beginning. This may be during production, in storage, transportation, or even before the actual use.

As $\gamma$ delivers an estimation of the earliest time-to-failure of such units, the operating period from 0 to $+\gamma$ is a failure free of such units. 


\section{ii) Cause of breakdowns following 3-P Weibull distribution}

Three types of break down cause are found to be following 3-P; maintenance, Stocking, and others. Figures 9, 10, and 11 represent probability density function ( $p d f)$, failure rate vs. time, and reliability vs. time, of stocking cause of failure.

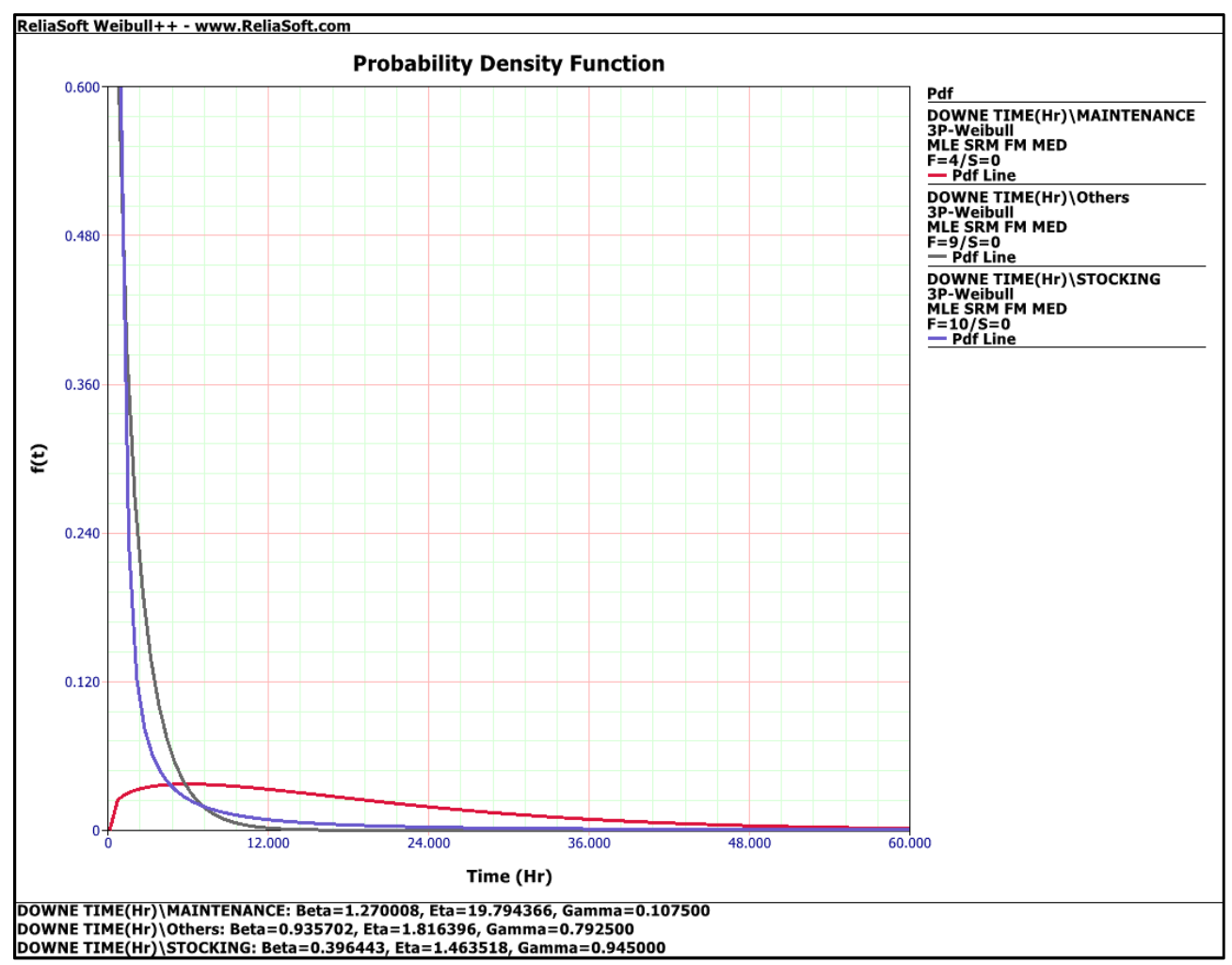

Figure 9: PDF function of the three causes of downtime following (3-P Weibull) Maintenance, Stoking and Others

It could be seen in figure 11 that maintenance $p d f$ (red line) which has the value of $\boldsymbol{\eta}=19.794366$ exhibits a different behaviour (shape) than the stocking and other causes $p d f$ that have $\mathbf{1}<\boldsymbol{\eta}<\mathbf{2}$. It is also noted that for the maintenance cause of failure; red curve, $\boldsymbol{\beta}<\mathbf{1}$ while stocking and other causes $\boldsymbol{\beta}>\mathbf{1}$. The characteristics of these cases are mentioned in "The Effect of beta on the pdf" subsection. 


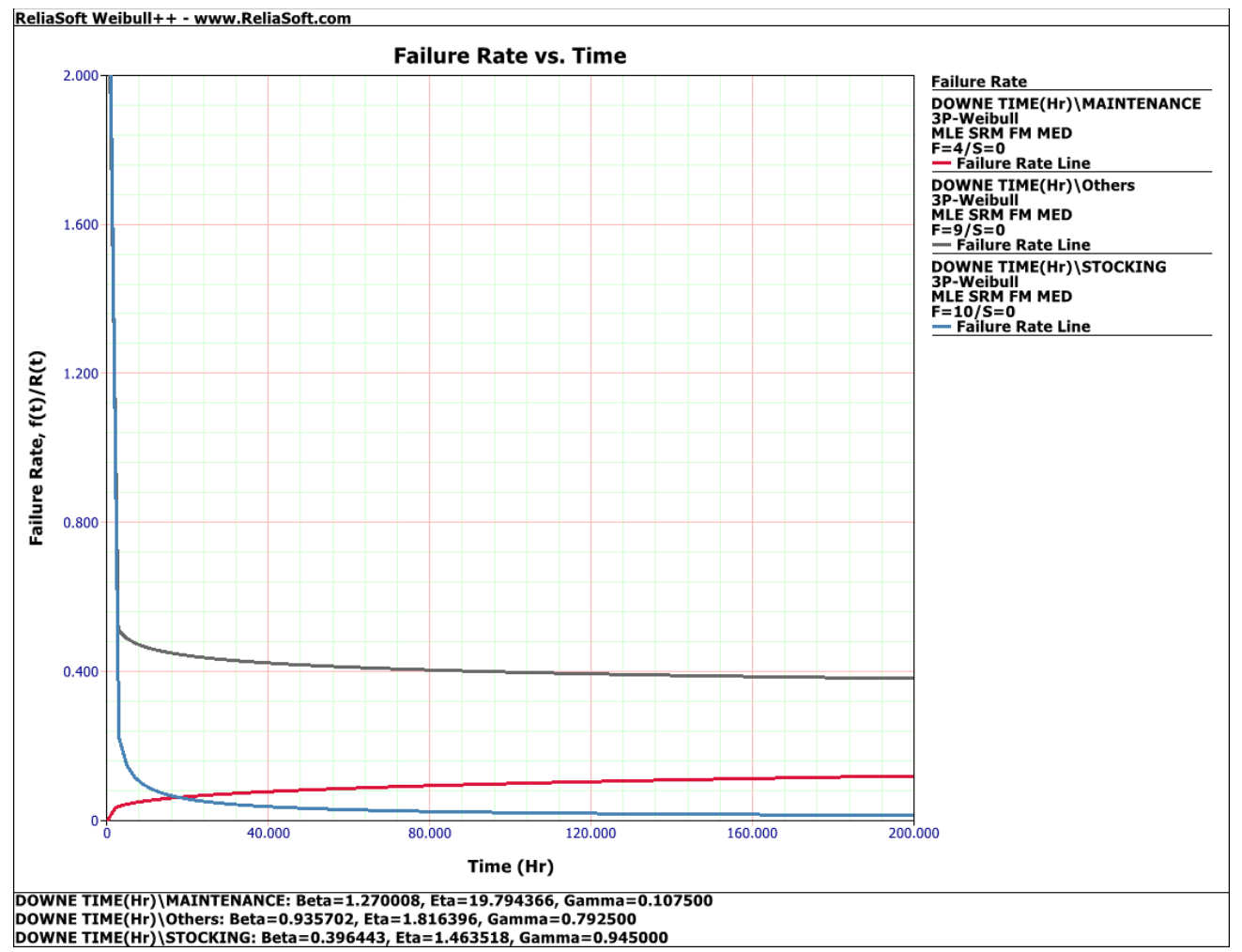

Figure 10: Failure rate vs. Time of the three causes of downtime following (3-P Weibull) Maintenance, stoking and others

As indicated in figure 10, downtime data with $\beta<1$ (other causes and stoking) exhibit a failure rate that decreases with time In case of down time due to maintenance where $\beta>1$, failure rate increases with time. This behaviour is previously explained in subsection (ii). 


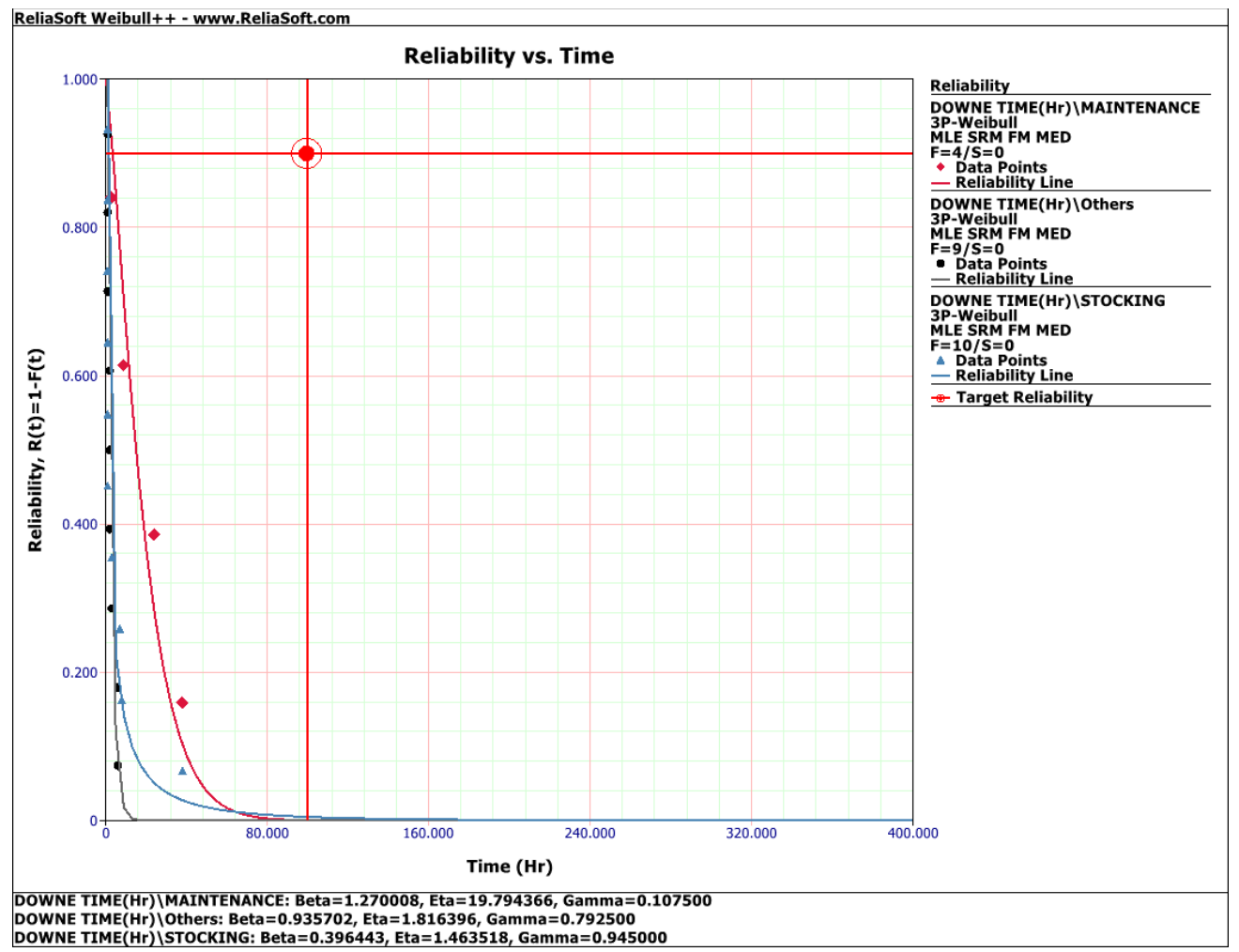

Figure 11: Reliability vs. Time of the three causes of downtime following (3-P Weibull) Maintenance, Stoking and Others

It could be seen in figure 11 that the reliability of stocking and other causes decreases sharply as $0<\beta<1$ for both of them; 0.396443 and 0.935702 , respectively. While the decrease of maintenance reliability curve is less sharp as $\beta=1.27(>1)$ according to the characteristics previously mentioned in section 4.2.2 (i).

\subsubsection{Gamma Distribution}

i) Gamma distribution characteristics Gamma distribution has 2 parameters; scale parameter $(\mathrm{k})$ and shape parameter $(\mu)$. Figure 12 shows probability density function ( $p d f$ ) of Gamma distribution for $k>1$. 


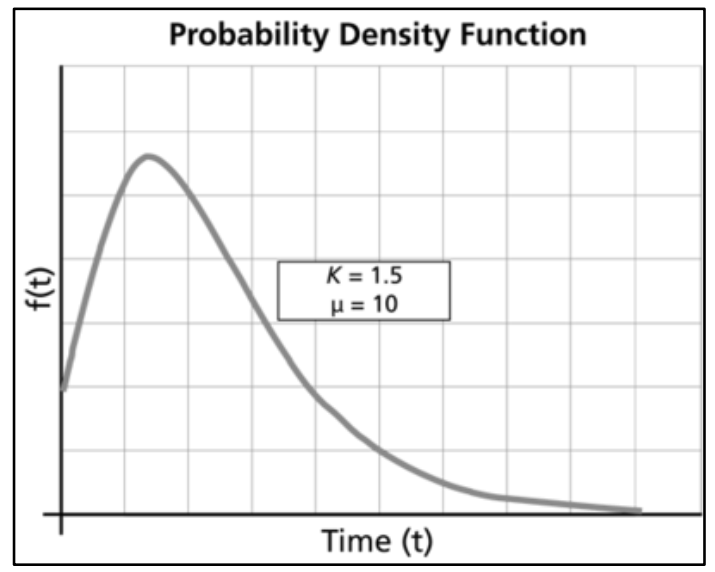

Figure 12: Probability density Function ( $p d f)$ of Gamma distribution for $k>1$ [27]

In case of $\mathrm{k}=1$ Gamma becomes the exponential distribution, [30, and 35].

As $f(T) \rightarrow \frac{1}{e^{\mu^{\mu}}}$, then as $t \rightarrow \infty, f(t) \rightarrow 0$, thus the $p d f$ decreases monotonically and is convex.

As $\lambda(t)=\frac{1}{e^{\mu}}, \lambda(t)$ is constant, then the mode does not exist.

In case of $(0<k<1)$ :

As $t \rightarrow 0, f(t) \rightarrow \infty$

As $t \rightarrow \infty, f(t) \rightarrow 0$

As $t \rightarrow \infty, \lambda(t) \rightarrow \frac{1}{e^{\mu}}$

Figures $(13,14$, and 15) exhibit the effect of K parameter on Gamma $p d f$.

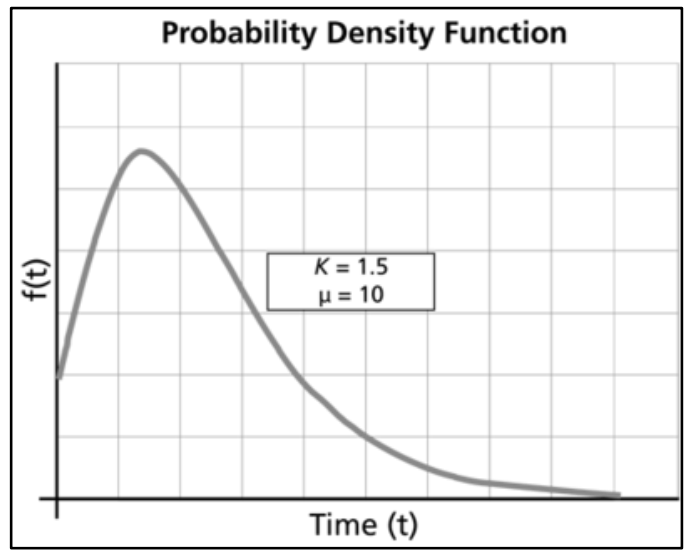

Figure13: Probability density Function $(p d f)$ of Gamma distribution for $k>1$

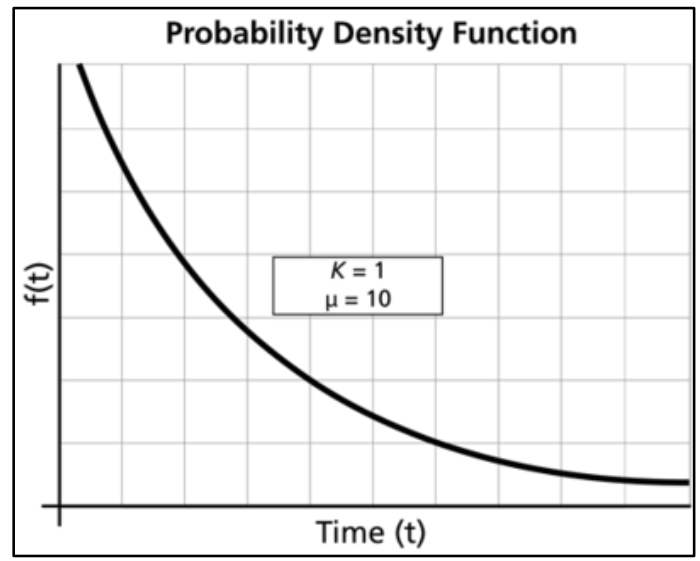

Figure 14: Probability density Function ( $p d f)$ of Gamma distribution for $k=1$ [27] 


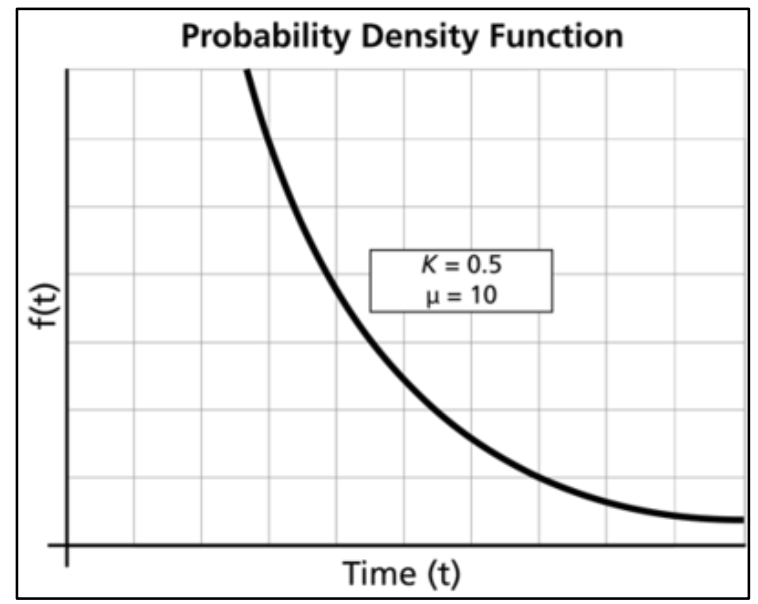

Figure 15: Probability density Function ( $p d f)$ of Gamma distribution for $0<\boldsymbol{k}<1$

It could be seen in figures 13,14 , and 15 that as $\mu$ increases, the $p d f$ becomes stretched out to the right and its height decreases, while preserving its shape. While as $\mu$ decreases, the $p d f$ shifts towards the left with increased height, and the mode does not exist.

\subsubsection{Generalized Gamma Distribution \\ i) Characteristics of Generalized Gamma}

As stated previously, the generalized gamma distribution contains other distributions as distinct cases built on the values of the parameters [36]. Figure16 exhibits the probability density function ( $p d f$ ) of Generalized Gamma distribution.

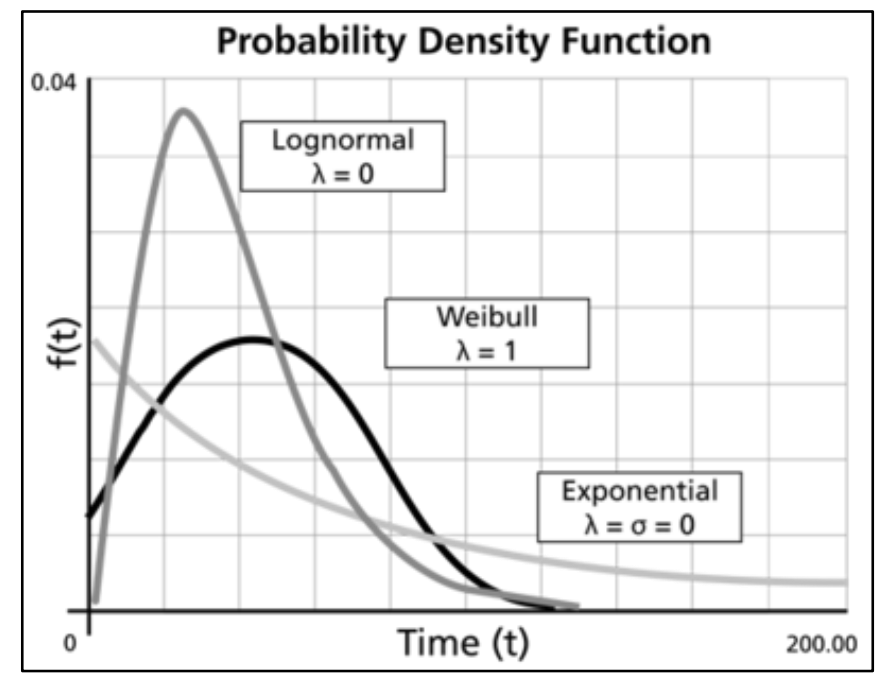

Figure16: Probability density Function ( $p d f)$ of Generalized Gamma distribution

\section{- Effects of the parameters $\sigma, \lambda$ and $\beta$}

From figure 19 the following could be extracted. The exponential distribution is a distinct case when $\lambda=1$ and $\sigma=1$. The lognormal distribution is a special case when $\lambda=0$. The gamma distribution 
is a distinctive case when $\lambda=\sigma$ and Weibull for $\sigma>\mathbf{1}, \boldsymbol{\sigma}=\mathbf{1}$, and $\sigma<\mathbf{1}, \boldsymbol{\beta}<\mathbf{1}, \boldsymbol{\beta}=\mathbf{1}$, and $\boldsymbol{\beta}>\mathbf{1}$, respectively).

\section{ii) Causes of breakdowns following Generalized Gamma distribution}

Three cause of downtime are found to follow Generalized Gamma distribution, these are; Electric, heating up, and tools change. Figure 17 exhibits the $(p d f)$ of the three causes of downtime, while figure 18 represents failure rate vs. Time of electric downtime causes and figure 19 shows Reliability vs. Time of Electric downtime.

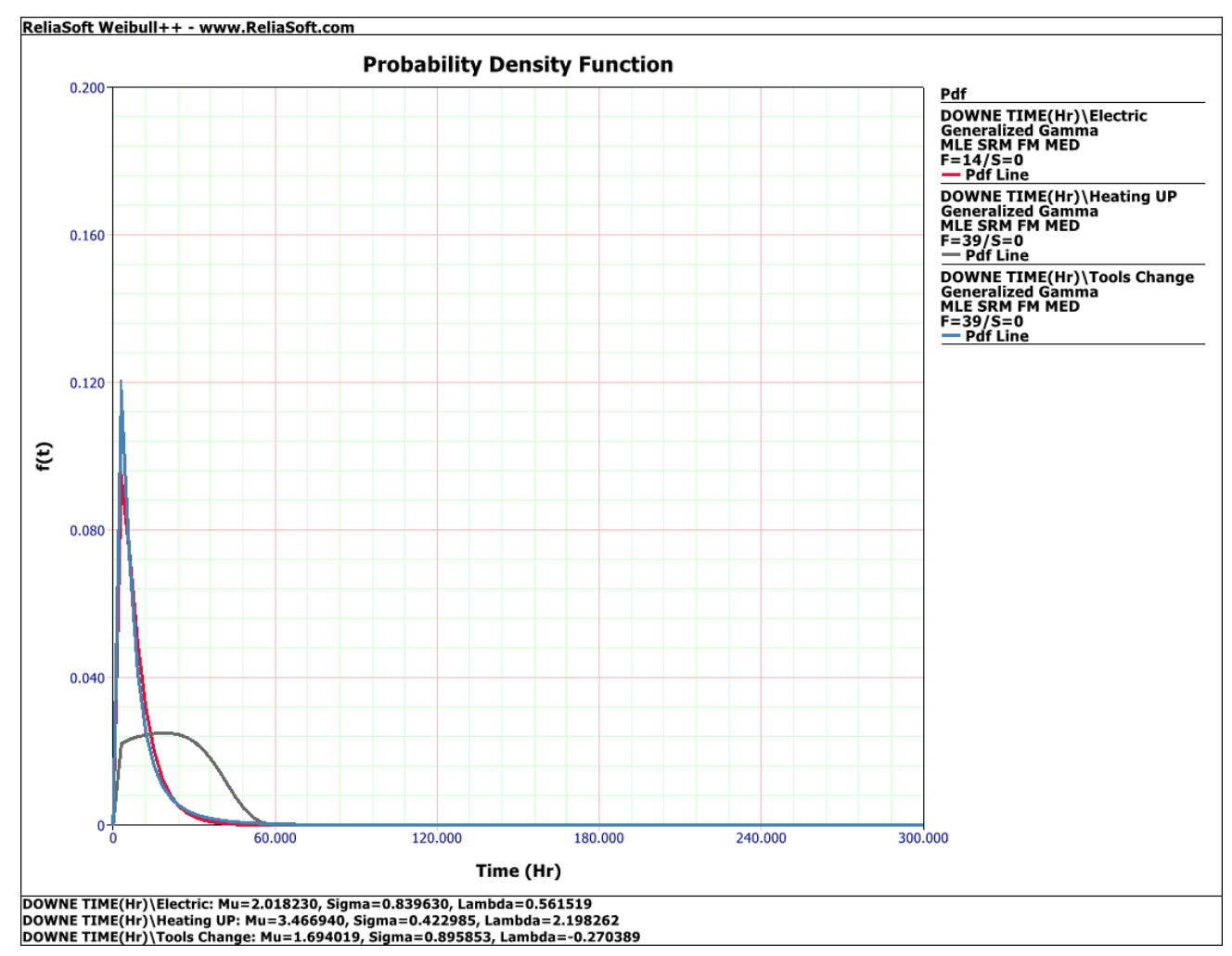

Figure 17: Probability density Function ( $p d f)$ of the three causes of downtime following (Generalized Gamma) electric, heating up, and tools change 


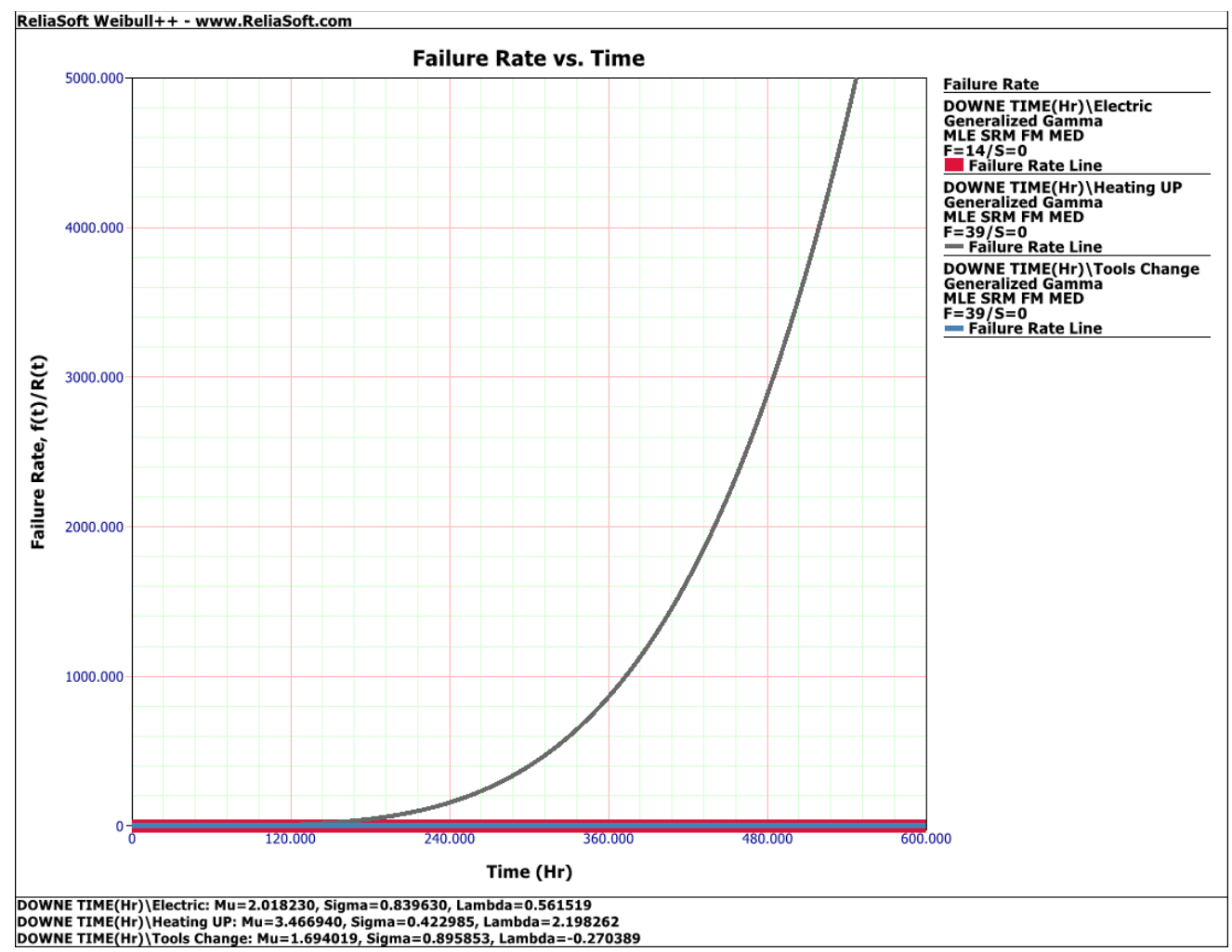

Figure 18: Failure rate vs. Time of Electric downtime, (generalized gamma distribution)

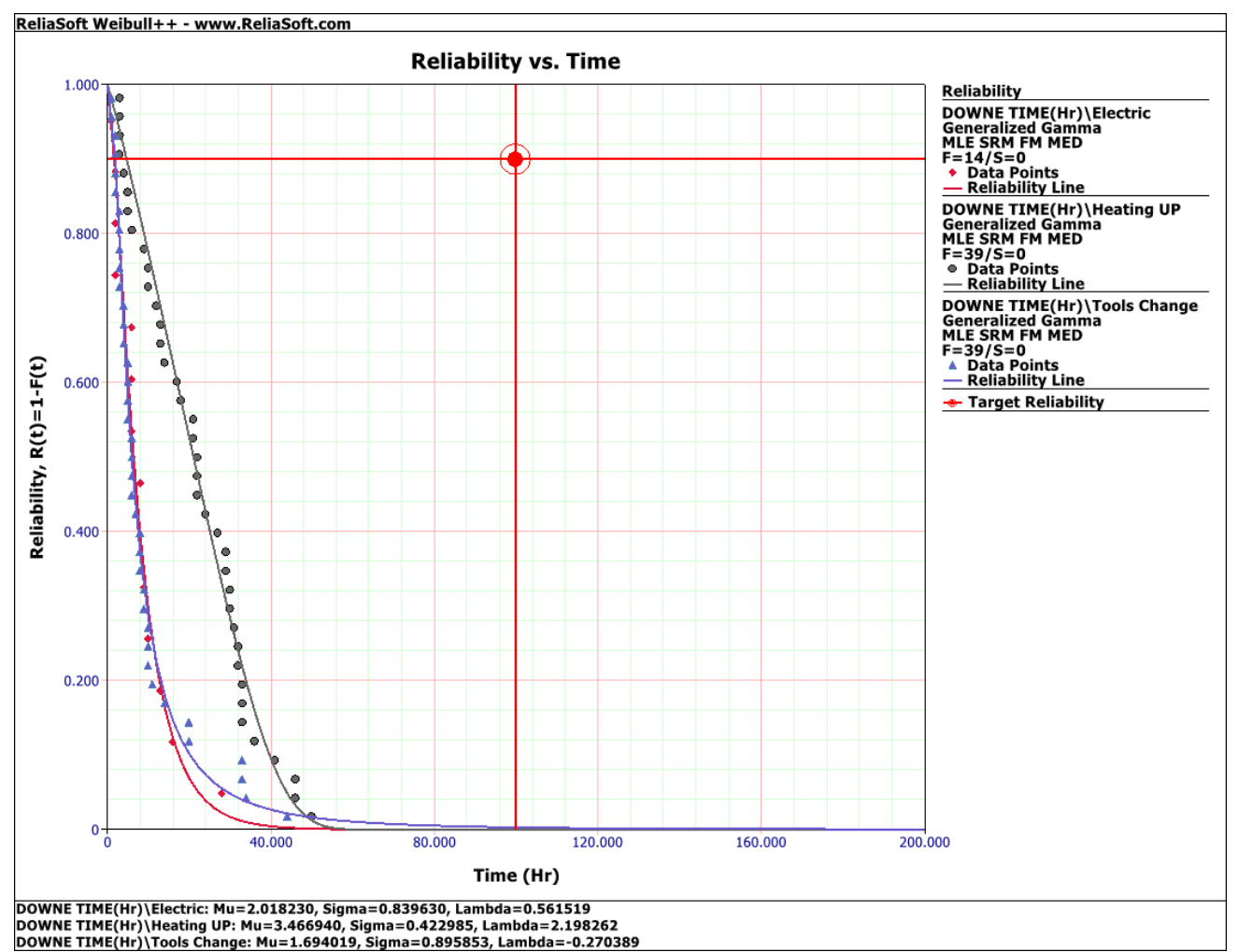

Figure 19: Reliability vs. Time of Electric downtime, of the three causes of downtime following (Generalized Gamma) electric, heating up, and tools change 
From figure 17 it could be seen that electric and tool changes have similar pdf behaviour where $\mu \mathrm{E}=$ $2.018, \mu \mathrm{TC}=1.694)$. Both have very close values of $\left.\sigma ; \sigma_{\mathrm{E}}=0.8395, \sigma_{\mathrm{TC}}=0.8958\right)$. The values of $\lambda$ are 0.561519 and 0.27039 , respectively. As for heating up cause of down time the parameters 'values are $(\mu=$ $3.4669, \sigma=0.422955$, and $\lambda=2.198$ ).

\subsubsection{Loglogistic Distribution}

\section{i) Characteristics of the loglogistic Distribution}

- Effects of the shape parameter

For $>1$ :

- $f(t)$ Decreases monotonically and is convex. Mode and mean do not exist.

For $\sigma=1$ :

- $f(t)$ Decreases monotonically and is convex. Mode and mean do not exist.

- As $t \rightarrow 0, f(t) \rightarrow \frac{1}{\sigma e^{\frac{\mu}{\sigma}}}$

- As $t \rightarrow 0, \lambda(t) \rightarrow \frac{1}{\sigma e^{\frac{\mu}{\sigma}}}$

For $0<\sigma<1$

- The shape of the loglogistic distribution is actual like to that of the lognormal distribution and the Weibull distribution.

- The $p d f$ starts at zero, increases to its mode, and decreases subsequently.

- As $\mu$ increases, while $\sigma$ is reserved similar, the $p d f$ becomes stretched out to the right and its height decreases, while preserving its shape.

- As $\mu$ decreases, while $\sigma$ is kept the same, the $p d f$ gets pushed in towards the left and its height increases. Figures 20, 21 and 22 exhibit the pdf, failure rate vs. time, and reliability vs. time of machine down time that follows Loglogistic distribution. 
ReliaSoft Weibull++ - www.ReliaSoft.com

Figure 20: Probability density function of Loglogistic distribution

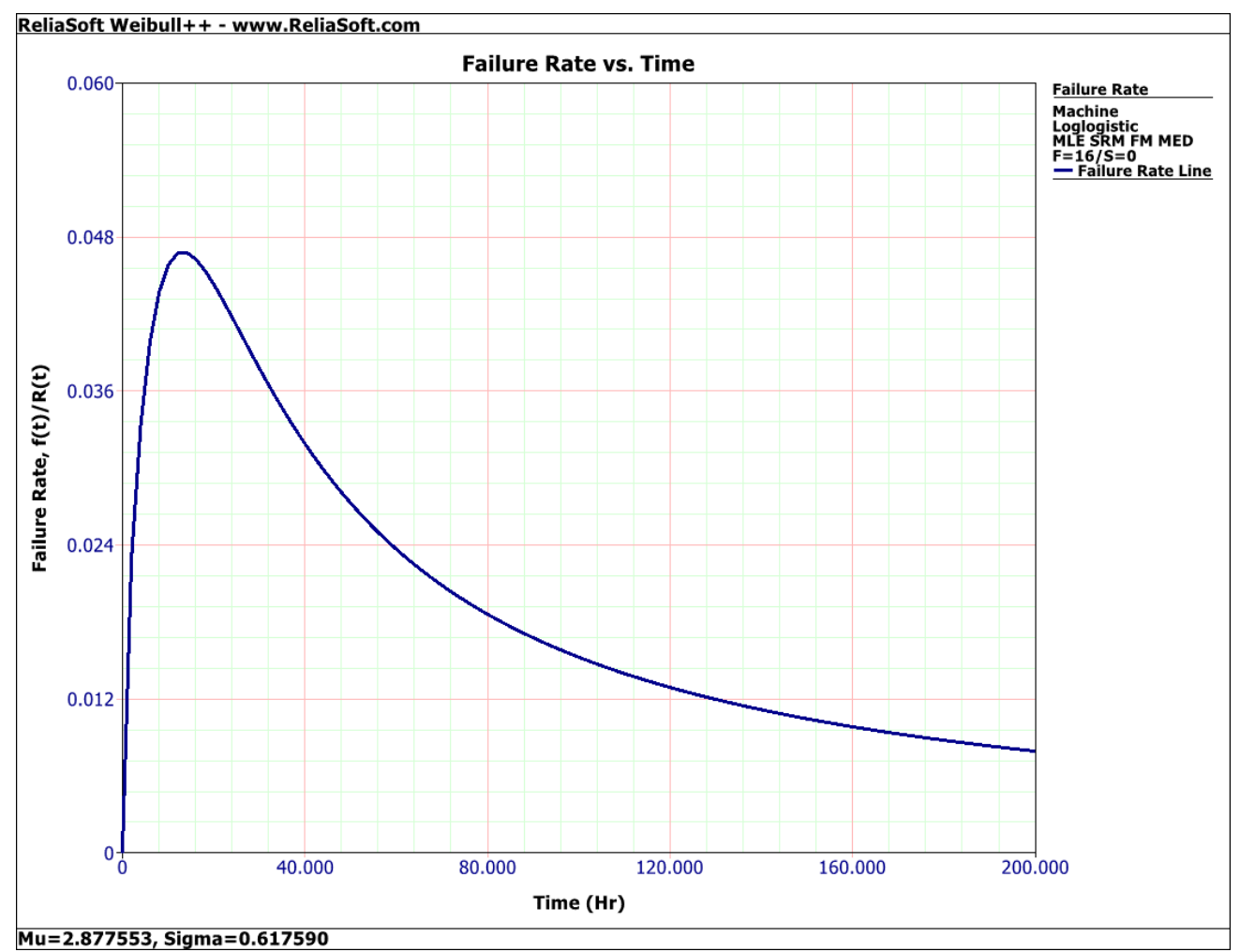

Figure 21: Failure rate vs. Time of machine downtime, (Loglogistic distribution) 
From figure 21 it can be deducted that the failure rate increases rapidly to 0.046 (in about $12 \mathrm{hr}$ ), then it decreases gradually to 0.0312 after $40 \mathrm{hrs}$, and finally reaches 0.046 after 200 hours.

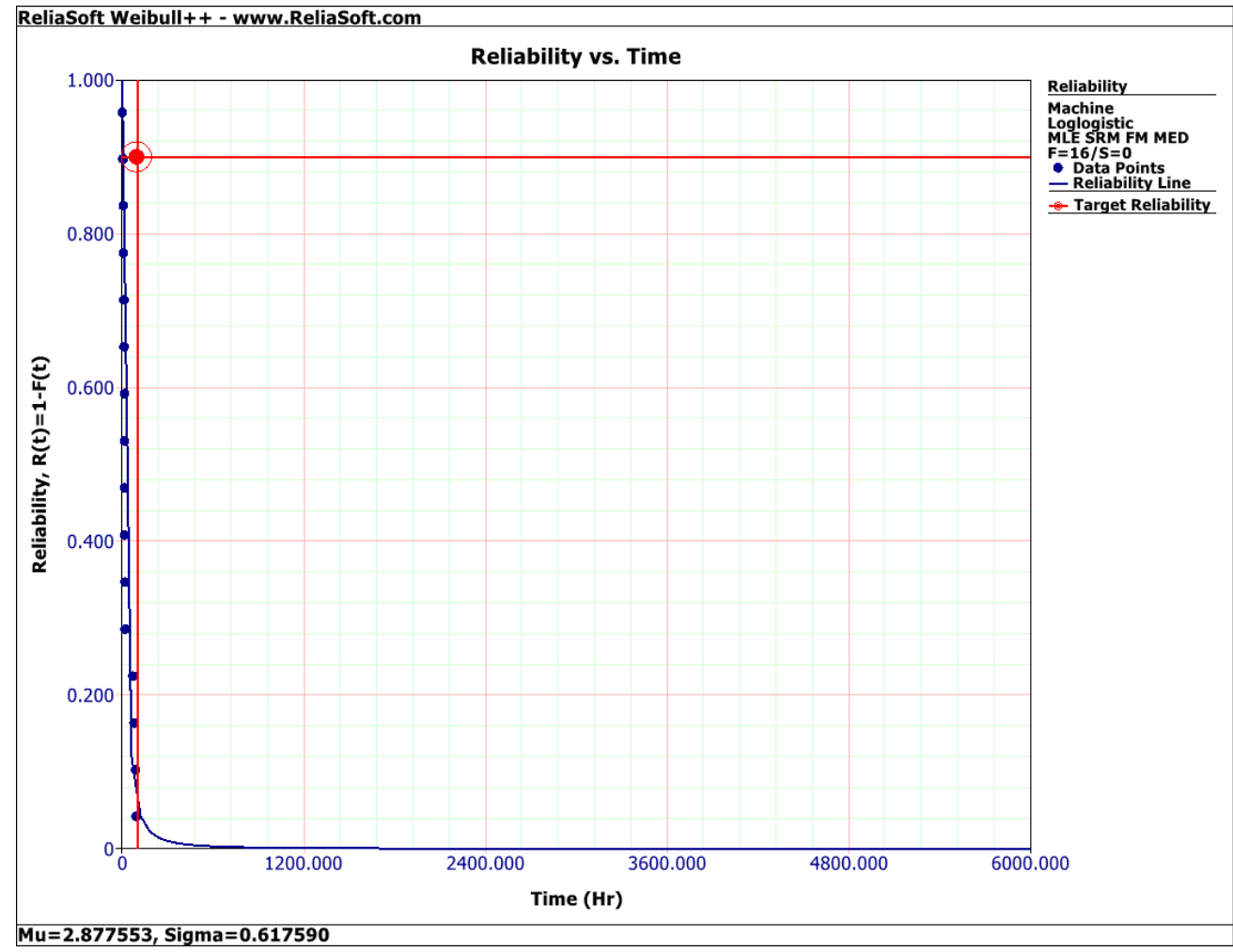

Figure 22: Reliability vs. Time of Machine downtime, (Loglogistic distribution)

From figure 22 it is noticed that the reliability of the machine had decreased very rapidly to $4 \%$ in 240 hrs period.

\subsubsection{Exponential Distribution}

\section{i) Characteristics of the2-Parameters exponential distribution}

Exponential distribution is used for modelling the behaviour of items with a constant failure rate. It is easy to manipulate as its mathematical form is quite simple with one parameter and it has no shape parameter. This fact may lead to the use of this model in conditions where it is not appropriate [37].

\section{- Effect of $\lambda$ and $\gamma$ on the exponential $p d f$}

Figure 23 exhibits the effect of the failure rate $\lambda$ on the "exponential distribution" $p d f$ 


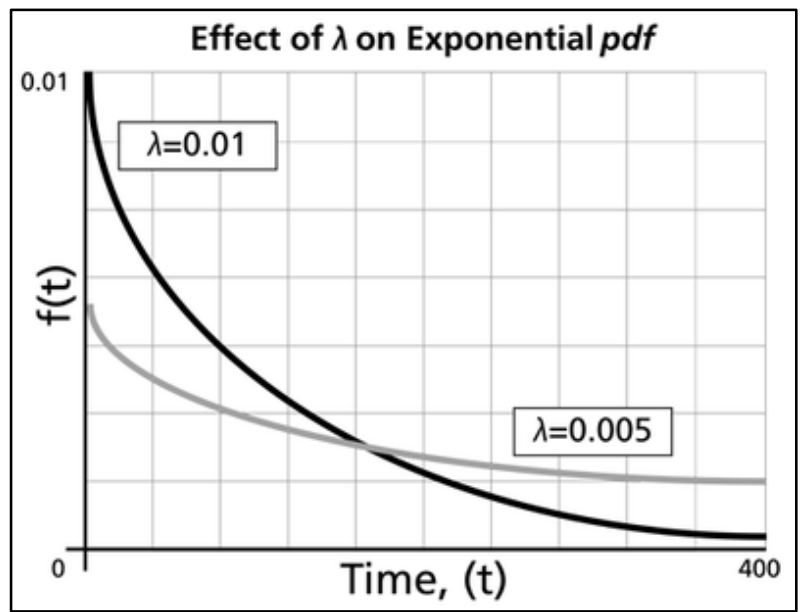

Figure 23: Effect of $\lambda$ on Exponential $p d f$ [27]

It could be seen in figure 23 that the exponential $p d f$ is always convex and is stretched to the right as $\lambda$ decreases in value and the value of the $p d f$ function is always equal to the value of $\lambda$ at $t=$ $0($ or $t=\gamma)$. As $t \rightarrow \infty, f(t) \rightarrow 0$. The scale parameter is $\frac{1}{\lambda}=\bar{T}-\gamma=m-\gamma$.

Figure 24 illustrates the $p d f$ of Air Drop cause of failure that follows 2P- Exponential distribution.

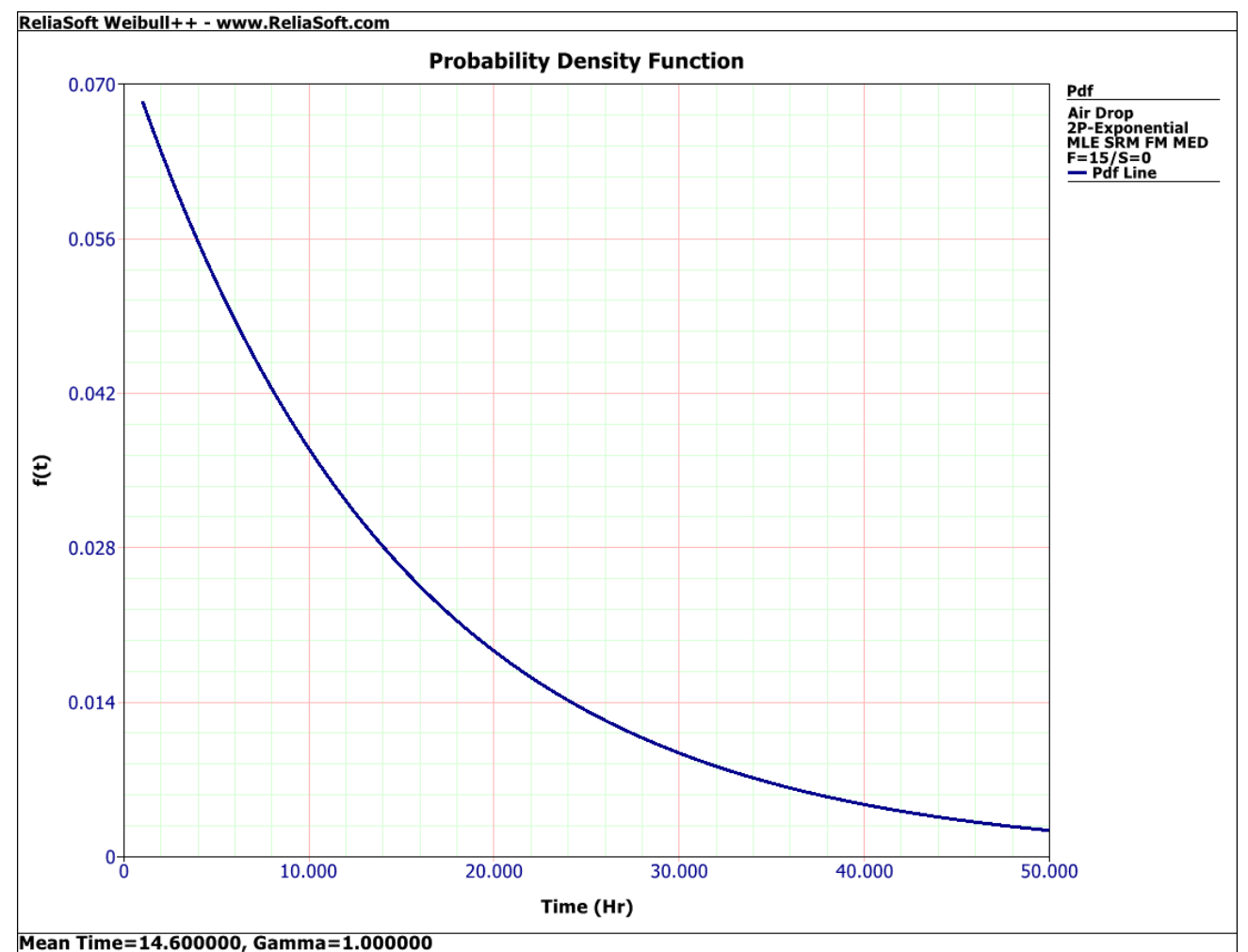

Figure 24: $P D F$ of Air Drop cause of failure (2P- Exponential)

As explained in the previous section; Effect of $\lambda$ and $\gamma$ on the exponential $p d f$, the air drop $p d f$ curve; figure 24, does not begin at time (0) but is shifted to the right at ( $t=1$ as $\gamma=1$ at this case). 


\section{- Effect of $\lambda$ and $\gamma$ on the exponential reliability function}

- The 1-parameter exponential reliability function starts at the value of $100 \%$ at $\mathrm{t}=0$, declining afterward monotonically.

- The 2-parameter exponential reliability function remnants at the value of $100 \%$ for $t=0$ up to $t=$ $\gamma$, and declining afterward.

- When $t \rightarrow \infty$, the Reliability $\rightarrow 0$

- The reliability for task with duration of one MTTF $\left(=\frac{1}{\lambda}\right)$ always equals $36.79 \%$. This means that only $36.8 \%$ of the task will be completed successfully. In other words, only $36.8 \%$ of the equipment undertaking this mission will survive their mission.

Figure 25 shows the effect of $\lambda$ on the reliability of air drop cause of failure.

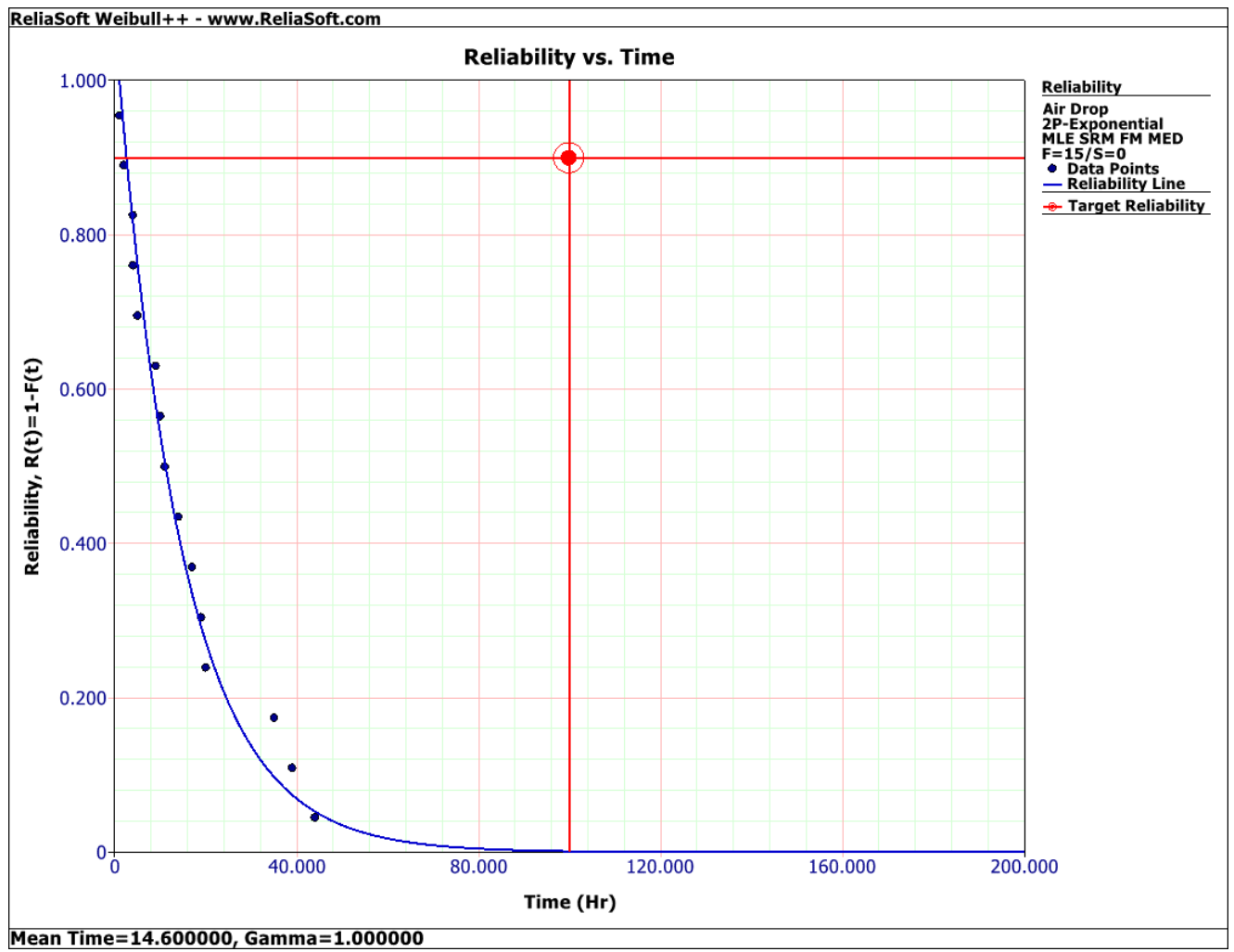

Figure 25: Reliability of Air Drop cause of failure (2P- Exponential)

\section{- Effect of $\lambda$ and $\gamma$ on the Failure Rate Function}

- One-parameter exponential failure rate function is constant and starts at $t=0$.

- Two-parameter exponential failure rate function remainders at the value of 0 for $t=0$ up to $=$ $\gamma$, and then preserves at the constant value of $\lambda$. Figure 26 displays the Effect of $\lambda$ and $\gamma$ on the Failure Rate Function. 


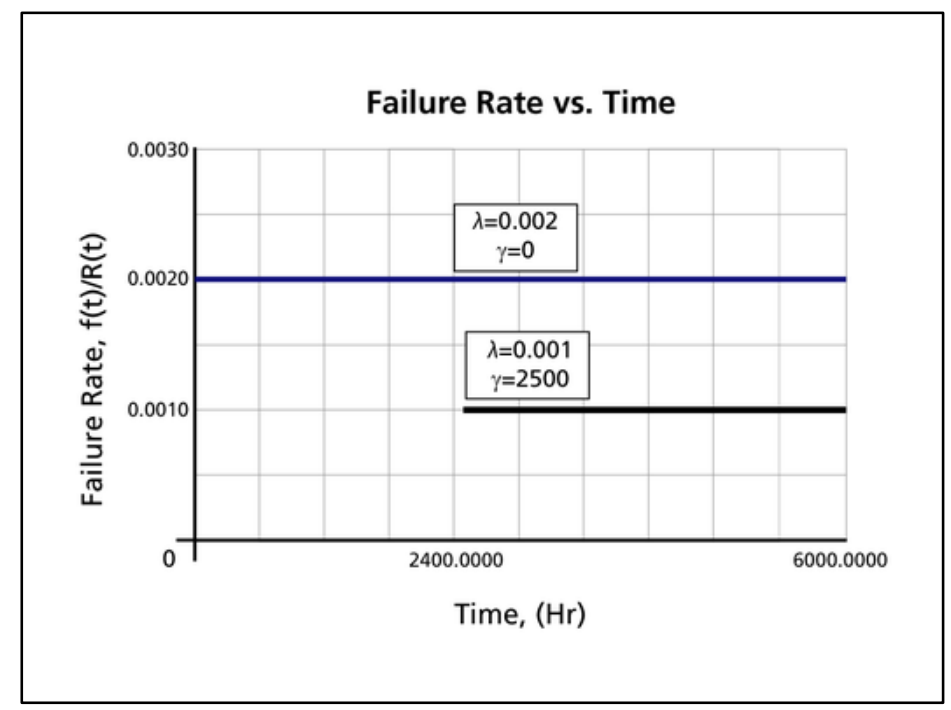

Figure 26: Effect of $\lambda$ and $\gamma$ on the Failure Rate Function

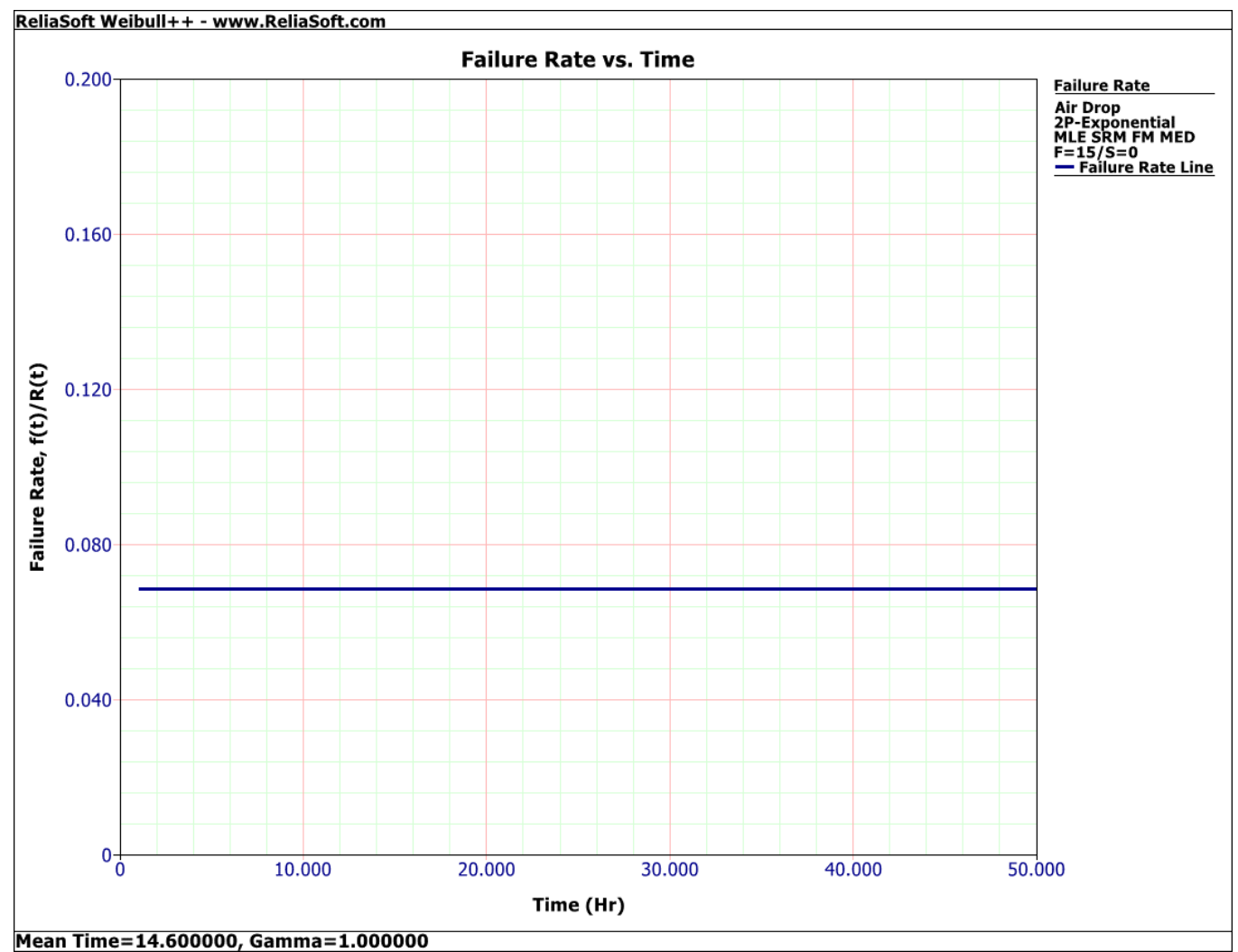

Figure 27: Failure rate vs. time of Air Drop cause (2P- Exponential) 
Figure 27 shows that failure rate of Air Drop cause of failure that follows 2-p exponential distribution is constant and identical to the characteristics in reference [27] shown in figure 26.

Table 3 presents a summary of different fitted distributions and their parameters for the different causes of defects for Coldair Engineering Company for refrigerators and air conditioners in Sudan, while table 4 summarizes the different parameters.

Table 3: Summary of different distributions and their parameters for the different causes of defects

\begin{tabular}{|c|c|c|c|c|}
\hline $\begin{array}{l}\text { Cause of Down } \\
\text { Time }\end{array}$ & Distribution & \multicolumn{3}{|l|}{ Parameters Value } \\
\hline \multirow[t]{2}{*}{ NSE POWER OFF } & \multirow{2}{*}{ Lognormal } & LMean & LStd & \\
\hline & & 2.58166532542109 & 0.99576817682367 & \\
\hline & & Beta & Eta & Gamma \\
\hline OTHERS & 3P-Weibull & 0.935701723595922 & 1.81639643746557 & 0.792499999999999 \\
\hline STOCKING & 3P-Weibull & 0.396442512537193 & 1.46351753807986 & 0.944999999999998 \\
\hline MAINTENANCE & 3P-Weibull & 1.27000818461819 & 19.7943664820936 & 0.1075 \\
\hline \multirow[t]{5}{*}{ ELECTRIC } & \multirow[t]{2}{*}{ Gamma } & $\mathrm{Mu}$ & $\mathrm{K}$ & \\
\hline & & 1.6767141546887 & 1.56268023405527 & \\
\hline & \multirow{2}{*}{ G-Gamma } & Mean & Std & Lambda \\
\hline & & 2.01822965270241 & 0.839630327183401 & 0.561518942443327 \\
\hline & & Mean & Std & Lambda \\
\hline $\begin{array}{l}\text { TOOLS } \\
\text { CHANGE }\end{array}$ & G-Gamma & 3.46693976881869 & 0.422985396246437 & 2.19826185801417 \\
\hline HEATING UP & G-Gamma & 1.69401933700553 & 0.895853121581676 & 0.270389116103458 \\
\hline \multirow[t]{2}{*}{ MACHINE } & \multirow{2}{*}{ Loglogistic } & $\mathrm{Mu}$ & Sigma & \\
\hline & & 2.87755257897385 & 0.617590048768441 & \\
\hline \multirow[t]{2}{*}{ AIR DROP } & \multirow{2}{*}{$\begin{array}{l}2 \mathrm{P}- \\
\text { Exponentional }\end{array}$} & Lambda & Gamma & \\
\hline & & 0.0684931506849315 & 1 & \\
\hline
\end{tabular}


Table 4: Summary of the parameters of different fitted distributions

\begin{tabular}{|c|c|c|c|}
\hline Distribution & Parameters & & \\
\hline \multirow[t]{2}{*}{ Lognormal } & LMean & LStd & \\
\hline & $\begin{array}{l}\text { Mean of the log of the } \\
\text { distribution }(\dot{\mu})\end{array}$ & $\begin{array}{l}\text { standard deviation of } \\
\text { the log of the } \\
\text { distribution }(\sigma)\end{array}$ & \\
\hline \multirow[t]{2}{*}{ 3P-Weibull } & Beta & Eta & Gamma \\
\hline & $\begin{array}{r}\text { shape parameter } \\
\boldsymbol{\beta}\end{array}$ & $\begin{array}{c}\text { scale parameter } \\
\boldsymbol{\eta}\end{array}$ & $\begin{array}{l}\text { Location parameter } \\
\qquad \gamma\end{array}$ \\
\hline \multirow[t]{2}{*}{ Gamma } & $\mathrm{Mu}$ & $\mathrm{K}$ & \\
\hline & Shape parameter $(\mu)$ & scale parameter $(K)$ & \\
\hline \multirow[t]{2}{*}{ G-Gamma } & Mean & Std & Lambda \\
\hline & shape parameter $(\mu)$ & shape parameter $(\sigma)$ & scale parameter $(\lambda)$ \\
\hline \multirow[t]{2}{*}{ Loglogistic } & $\mathrm{Mu}$ & Sigma & \\
\hline & scale parameter $(\mu)$ & shape parameter $(\sigma)$ & \\
\hline \multirow[t]{2}{*}{ 2P-Exponentional } & Lambda $(\lambda)$ & $\operatorname{Gamma}(\gamma)$ & \\
\hline & Rate parameter & location parameter & \\
\hline
\end{tabular}

\section{Conclusions}

To predict the reliability performance of an item or component, available life data; down time or failure time is analyzed and fitted to a novel statistical distributions using Weibull++ ALTA Software. For the case under study downtime data were fitted to different distributions and the parameters are estimated by two methods using both MLE and RRX and it gave the same results. The resulted distributions are then tested using K-S goodness of fit test, Correlation Coefficient (CC) test and Likelihood Value (LHV) test. Based on the three rankings; resulted from the three mentioned tests, and on weights assigned to the individual criteria, an aggregate criterion (DESV) is applied to choose the best-fitting probability distribution. In this study, the used weights are the default values of weights selected by the software "Weibull++/ ALTA" manufacturer.

\section{Specific conclusions:}

1. In the case under study the causes of downtime are:

- Maintenance, Stoking and Others which were found to follow 3-P Weibull.

- Air drop following 2-P exponential distribution

- Electric, Heating up, and Tools change causes following Generalized Gamma

- NSE power off found to follow the log normal distribution

- Machine following Log-logistic 
2. The three cause following 3-P Weibull, maintenance $p d f$ which has $(=19.794366$ and $\beta<1)$ exhibits a different behaviour (shape) than the stocking and other causes $p d f$ that have $(1<\eta<2$ and $\beta>1)$. This different behaviour also applies for failure rate and reliability as for $\beta<1$ (stoking and other causes) the failure rate decreases with time and decreases sharply. In case of $\beta>1$ (maintenance), failure rate increases with time while the decrease in reliability curve is less sharp.

3. Weibull distribution is a special case of Generalized Gamma when $\lambda=1$ and $\beta=\frac{1}{\sigma} \eta=\exp (\mu)$

4. Downtime data with $\beta<1$ exhibit a failure rate that decreases with time, while that with $\beta=1$ have a constant failure rate (consistent with the exponential distribution) and populations with $\beta>1$ have a failure rate that increases with time. This means that all three life stages of reliability can be modeled with the Weibull distribution.

\section{Acknowledgment}

I would like to thank the Department of Mathematics and Prince Sattam bin Abdulaziz University for their kind support for my research work and I am very proud to be a faculty member in this brilliant institution.

\section{REFERENCES}

[1] M. Khodabin and A.R. Ahmadabadi, "Some properties of generalized gamma distribution", Mathematical Sciences 2010, Vol. 4, No. 1

[2] Jaggia S. "Specification tests based on the heterogeneous generalized gamma model of duration: with an application to Kennan's strike data,", Journal of Applied Econometrics, 1991, 6, 169-180.

[3] Yamaguchi K. "Accelerated failure-time regression models with a regression model of surviving fraction: an application to the analysis of permanent employment in Japan", Journal of the American Statistical Association, 1992, 87, 284-92

[4] Allenby G.M., Leone R.P., Jen L." A Dynamic model of purchase timing with application to direct marketing," Journal of the American Statistical Association, 1999, 94, 365-74

[5] Emad E. Elmahdy, "Modeling Reliability Data with Finite Weibull or Lognormal Mixture Distributions", Appl. Math. 2017, Inf. Sci.11, No. 4, 1081-1089.

[6] MeryamKrit, "Goodness-of-fit tests in reliability: Weibull distribution and imperfect maintenance models”. General Mathematics [math.GM]. Ph. D. thesis. University of Grenoble, 2014

[7] F.P.A. Coolen, "Parametric probability distributions in reliability", Contribution to Wiley Encyclopedia of Quantitative Risk Analysis and Assessment, 2008 https://doi.org/10.1002/9780470061596.risk0466

[8] Mohieddine Rahmouni and Ayman Orabi "A generalization of the exponential-logarithmic distribution for reliability and life data analysis" Life Cycle Reliability and Safety Engineering 2018, 7:159-171 https://doi.org/10.1007/s41872-018-0049-5

[9] Vito Ricci, "Fitting Distributions with R", R: A language and environment for statistical computing. RDevelopment Core Team (2004). R-Foundation for Statistical Computing, Vienna, Austria, February 2005. ISBN 3-900051-07-0, http://www.r-project.org 
[10] Adegbulugbe, A. O., "Balancing the Acts in the Power Sector: The Unfolding Story of Nigeria Independent Power Projects", 2007, 27th USAEE/IAEE North American Conference,.

[11] Denson W, "Rebuttal to: A critique of the Reliability Analysis Centre Failure Rate Model for Plastic Encapsulated Microcircuits", IEEE Trans Reliability, 1998,Vol.47, Issue 4, PP.419-424.

[12] Y. S. Thakur and D. K. Sakravdia, "Reliability Prediction Methods for Electronic Devices and Systems- A Review", International Journal of Applied Engineering Research, 2018 Vol.13, Issue17, PP. 13063-13069. https://dx.doi.org/10.37622/IJAER/13.

[13] Hongqin Fan, "Data Mining and Statistical Analysis of Construction Equipment Failure" 2012 29 $29^{\text {th }}$ ISARC, Eindhoven, Netherlands.

[14] Barabady, J. and Kumar, U., "Reliability Analysis of Mining Equipment: A Case Study of A Crushing Plant at Jajarm Bauxite Mine in Iran", Reliability Engineering and System Safety, 2008 Vol. 93, PP. 647-653. https://doi.org/10.1016/j.ress.2007.10.006

[15] AC Aneke, AO Odukwe and TO Onah, "Effect of Failure Rates on System Reliability of A Gas Turbine Power Plant", International Journal of Multidisciplinary Research and Development, 2017, Vol. 4; Issue 7, PP.68-73.

[16] Subhash Mathew, "Quality and Reliability Corner Optimal Inspection Frequency A Tool for Maintenance Planning/Forecasting", International Journal of Quality \& Reliability Management, 2004, Vol. 21, Issue.7, PP. 763-771. DOI 10.1108/02656710410549109

[17] R._Hesamamiri, M. M._Mazdeh, M. Jafari, “Measuring the reliability of knowledge management Instrument development and validation", Aslib Proceedings ,March 201465(5):484 - 502.

[18] Kuo W and ZuoMJ., "Optimal Reliability Modeling: Principles and Introduction to Maintenance and Reliability", Ambik Press Ltd, Benin, Nigeria, 2003.

[19] K. Krishnamoorthy, "Handbook of Statistical Distributions with Applications", Taylor \& Francis Group, 2006

[20] Bavuso S J., "Aerospace Applications of Weibull and Monte Carlo Simulation with Importance Sampling", IEEE, Annual Reliability and Maintainability Symposium, 1997.

[21] Kececioglu, Dimitri, Reliability Engineering Handbook, Prentice Hall, Inc., Englewood Cliffs, New Jersey, 1991, Vol. 1.

[22] Kececioglu, Dimitri, Reliability \& Life Testing Handbook, Prentice Hall, Inc., Englewood Cliffs, New Jersey, Vol. 1 and 2, 1993 and 1994.

[23] Afrah Abdullah ,Amina Ahmed, Taghreed mohmmed Ali, Afraa Abdulatif "A Comparison Study for the Methods of Estimating the Parameters of Weibull Distribution and its Role in the Reliability Applied Case in COLDAIR " final project report $2016 \underline{\mathrm{http}: / / \text { repository.sustech.edu/handle/123456789/17223 }}$

[24] Stephens, M. A. "Asymptotic Results for Goodness-of-Fit Statistics with Unknown Parameters", Annals of Statistics, 1976, 4, pp. 357-369. 
[25] Lee E T and Wang J W., "Statistical Methods for Survival Data Analysis", John Wiley \& Sons Inc, 3rd Edition, 2003. https://doi.org/10.1002/0471458546

[26] Crow, E. L. and Shimizu, K. (Ed.). “Lognormal Distributions: Theory and Applications”. New York: Dekker, 1988.

[27] Abernethy, Dr. Robert B., “The New Weibull Handbook”. Houston: Gulf Publishing Company, 2008.

[28] Bryan Dodson “Weibull Analysis" Paperback - December 1, 1994

[29] Barreto-Souza W, de Morais AL, Cordeiro GM, “The Weibull geometric distribution". J Stat ComputSimul, 2011, 81(5):645-657.

[30] Lawless, J.F., “Statistical Models and Methods for Lifetime Data, John Wiley \& Sons, Inc., New York, 1982.

[31] Rigby, R. A., Stasinopoulos, D. M., Heller, G. Z., and De Bastiani, F. Distributions for modeling location, scale, and shape: Using GAMLSS in R, Chapman and Hall/CRC. 2019 An older version can be found in http://www.gamlss.com/.

[32] Federico Reghenzani , Giuseppe Massari , Luca Santinelli , William Fornaciari “Statistical power estimation

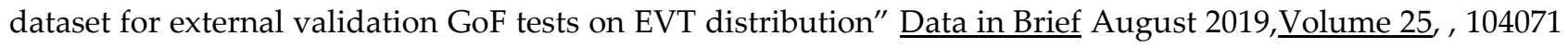
https://doi.org/10.1016/j.dib.2019.104071

[33] Steven J. Fletcher Data Assimilation for the Geosciences. Copyright (c) 2017 Elsevier Inc. http://dx.doi.org/10.1016/B978-0-12-804444-5.00004-0

[34] Zuzana Obertová, Alistair Stewart and Cristina Cattaneo Statistics and Probability in Forensic Anthropology. 2020 Elsevier Inc. https://doi.org/10.1016/B978-0-12-815764-0.00011-3 \#

[35] Firuz Kamalov and Dmitry Denisov "Gamma distribution-based sampling for imbalanced data" Knowledge-Based Systems, November 2020, https://doi.org/10.1016/j.knosys.2020.106368

[36] O. Gomes C. Combes and A. Dussauchoy "Parameter estimation of the generalized gamma distribution" Mathematics and Computers in Simulation, 2008, 79, 955-963. https://doi.org/10.1016/j.matcom.2008.02.006

[37] Tahani A. Abushal "Parameter estimation of Weibull-Exponential distribution under Type-I hybrid censored sample" Journal of King Saud University - Science, October 2019, Volume 31, Issue 4, Pages 1431-1436. https://doi.org/10.1016/j.jksus.2018.02.004 
Appendix A: DESV Results of Down time fitted Data using MLE Method

\begin{tabular}{|c|c|c|c|c|c|c|c|c|c|c|c|c|c|c|c|c|c|}
\hline DT & Distribution & RAVGOF & RAVPLOT & RLKV & DESV & ROF & Distribution & RAVGOF & RAVPLOT & RLKV & DESV & ROF & Distribution & RAVGOF & RAVPLOT & RLKV & DESV \\
\hline \multirow{11}{*}{ 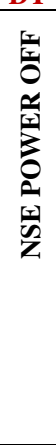 } & $\begin{array}{r}1 \mathrm{P}- \\
\text { Exponentional }\end{array}$ & 3 & 3 & 4 & 350 & \multirow{11}{*}{ 总 } & $\begin{array}{r}1 \mathrm{P}- \\
\text { Exponentional }\end{array}$ & 10 & 9 & 8 & 890 & \multirow{11}{*}{ 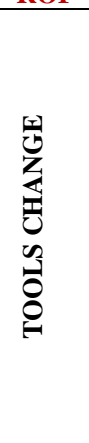 } & $\begin{array}{r}1 \mathrm{P}- \\
\text { Exponentional }\end{array}$ & 6 & 6 & 8 & 700 \\
\hline & $\begin{array}{r}2 \mathrm{P}- \\
\text { Exponentional }\end{array}$ & - & - & - & - & & $\begin{array}{r}2 \mathrm{P}- \\
\text { Exponentional }\end{array}$ & 8 & 7 & 1 & 440 & & $\begin{array}{r}2 \mathrm{P}- \\
\text { Exponentional }\end{array}$ & 5 & 5 & 1 & 300 \\
\hline & Normal & 6 & 7 & 7 & 660 & & Normal & 6 & 10 & 10 & 840 & & Normal & 10 & 10 & 10 & 1000 \\
\hline & Lognormal & 4 & 1 & 1 & 220 & & Lognormal & 9 & 8 & 6 & 740 & & Lognormal & 3 & 3 & 3 & 300 \\
\hline & 2P-Weibull & 1 & 4 & 3 & 230 & & 2P-Weibull & 2 & 3 & 5 & 360 & & 2P-Weibull & 7 & 7 & 7 & 700 \\
\hline & 3P-Weibull & - & - & - & - & & 3P-Weibull & 5 & 4 & 2 & 340 & & 3P-Weibull & 4 & 4 & 4 & 400 \\
\hline & Gamma & 2 & 5 & 2 & 230 & & Gamma & 3 & 1 & 4 & 330 & & Gamma & 8 & 8 & 6 & 700 \\
\hline & G-Gamma & - & - & - & - & & G-Gamma & 4 & 2 & 3 & 330 & & G-Gamma & 2 & 2 & 2 & 200 \\
\hline & Logistic & 7 & 6 & 6 & 640 & & Logistic & 1 & 5 & 9 & 540 & & Logistic & 9 & 9 & 9 & 900 \\
\hline & LogLogistic & 5 & 2 & 5 & 470 & & LogLogistic & 7 & 6 & 7 & 690 & & LogLogistic & 1 & 1 & 5 & 300 \\
\hline & Gumbel & 8 & 8 & 8 & 800 & & Gumbel & 11 & 11 & 11 & 1100 & & Gumbel & 11 & 11 & 11 & 1100 \\
\hline \multirow{12}{*}{ 先 } & Distribution & RAVGOF & RAVPLOT & RLKV & DESV & \multirow{12}{*}{ 窗 } & Distribution & RAVGOF & RAVPLOT & RLKV & DESV & \multirow{12}{*}{ 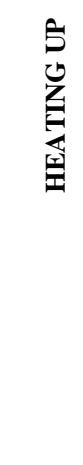 } & Distribution & RAVGOF & RAVPLOT & RLKV & DESV \\
\hline & $\begin{array}{r}1 \mathrm{P}- \\
\text { Exponentional } \\
\end{array}$ & 8 & 6 & 8 & 780 & & $\begin{array}{r}1 \mathrm{P}- \\
\text { Exponentional } \\
\end{array}$ & 4 & 4 & 7 & 550 & & $\begin{array}{r}1 \mathrm{P}- \\
\text { Exponentional }\end{array}$ & 10 & 10 & 9 & 950 \\
\hline & $\begin{array}{r}2 \mathrm{P}- \\
\text { Exponentional }\end{array}$ & 11 & 9 & 2 & 630 & & $\begin{array}{r}2 \mathrm{P}- \\
\text { Exponentional }\end{array}$ & 6 & 6 & 1 & 350 & & $\begin{array}{r}2 \mathrm{P}- \\
\text { Exponentional }\end{array}$ & 9 & 9 & 2 & 550 \\
\hline & Normal & 7 & 8 & 9 & 810 & & Normal & 9 & 9 & 8 & 850 & & Normal & 2 & 2 & 5 & 350 \\
\hline & Lognormal & 2 & 3 & 4 & 310 & & Lognormal & 2 & 2 & 4 & 300 & & Lognormal & 8 & 8 & 7 & 750 \\
\hline & 2P-Weibull & 6 & 4 & 7 & 630 & & 2P-Weibull & 5 & 5 & 6 & 550 & & 2P-Weibull & 5 & 5 & 3 & 400 \\
\hline & 3P-Weibull & 3 & 2 & 3 & 290 & & 3P-Weibull & - & - & - & - & & 3P-Weibull & - & - & - & - \\
\hline & Gamma & 4 & 5 & 6 & 510 & & Gamma & 7 & 7 & 5 & 600 & & Gamma & 7 & 6 & 4 & 540 \\
\hline & G-Gamma & 10 & 10 & 1 & 550 & & G-Gamma & 3 & 3 & 2 & 250 & & G-Gamma & 1 & 1 & 1 & 100 \\
\hline & Logistic & 5 & 7 & 10 & 770 & & Logistic & 8 & 8 & 9 & 850 & & Logistic & 3 & 3 & 6 & 450 \\
\hline & LogLogistic & 1 & 1 & 5 & 300 & & LogLogistic & 1 & 1 & 3 & 200 & & LogLogistic & 6 & 7 & 8 & 710 \\
\hline & Gumbel & 9 & 11 & 11 & 1020 & & Gumbel & 10 & 10 & 10 & 1000 & & Gumbel & 4 & 4 & 10 & 700 \\
\hline \multirow{12}{*}{ 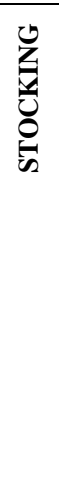 } & Distribution & RAVGOF & RAVPLOT & RLKV & DESV & \multirow{12}{*}{ 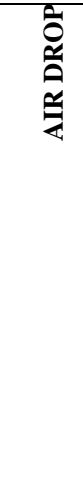 } & Distribution & RAVGOF & RAVPLOT & RLKV & DESV & \multirow{12}{*}{ 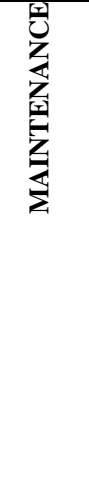 } & Distribution & RAVGOF & RAVPLOT & RLKV & DESV \\
\hline & $\begin{array}{r}1 \mathrm{P}- \\
\text { Exponentional }\end{array}$ & 9 & 7 & 8 & 830 & & $\begin{array}{r}1 \mathrm{P}- \\
\text { Exponentional }\end{array}$ & 1 & 5 & 6 & 390 & & $\begin{array}{r}1 \mathrm{P}- \\
\text { Exponentional }\end{array}$ & 5 & 1 & 6 & 510 \\
\hline & $\begin{array}{r}2 \mathrm{P}- \\
\text { Exponentional }\end{array}$ & 11 & 11 & 5 & 800 & & $\begin{array}{r}2 P- \\
\text { Exponentional }\end{array}$ & 3 & 6 & 1 & 230 & & $\begin{array}{r}2 \mathrm{P}- \\
\text { Exponentional }\end{array}$ & 11 & 11 & 2 & 650 \\
\hline & Normal & 2 & 8 & 10 & 660 & & Normal & 10 & 10 & 9 & 950 & & Normal & 6 & 3 & 9 & 720 \\
\hline & Lognormal & 6 & 2 & 3 & 410 & & Lognormal & 8 & 8 & 7 & 750 & & Lognormal & 1 & 2 & 7 & 410 \\
\hline & 2P-Weibull & 1 & 4 & 6 & 380 & & 2P-Weibull & 7 & 1 & 4 & 490 & & 2P-Weibull & 2 & 9 & 4 & 370 \\
\hline & 3P-Weibull & 7 & 1 & 1 & 340 & & 3P-Weibull & 4 & 4 & 2 & 300 & & 3P-Weibull & 3 & 8 & 3 & 350 \\
\hline & Gamma & 5 & 6 & 7 & 610 & & Gamma & 5 & 3 & 5 & 480 & & Gamma & 4 & 7 & 5 & 480 \\
\hline & G-Gamma & 10 & 10 & 2 & 600 & & G-Gamma & 6 & 2 & 3 & 410 & & G-Gamma & 10 & 10 & 1 & 550 \\
\hline & Logistic & 4 & 5 & 9 & 660 & & Logistic & 9 & 9 & 10 & 950 & & Logistic & 8 & 6 & 10 & 880 \\
\hline & LogLogistic & 3 & 3 & 4 & 350 & & LogLogistic & 2 & 7 & 8 & 550 & & LogLogistic & 7 & 4 & 8 & 720 \\
\hline & Gumbel & 8 & 9 & 11 & 960 & & Gumbel & 11 & 11 & 11 & 1100 & & Gumbel & 9 & 5 & 11 & 960 \\
\hline
\end{tabular}

